\title{
ON THE NATURE OF THE HERBIG B[e] STAR BINARY SYSTEM V921 SCORPII: GEOMETRY AND KINEMATICS OF THE CIRCUMPRIMARY DISK ON SUB-AU SCALES*
}

\author{
Stefan Kraus $^{1}$, Nuria Calvet ${ }^{1}$, Lee Hartmann ${ }^{1}$, Karl-Heinz Hofmann $^{2}$, Alexander Kreplin $^{2}$, \\ JoHn D. MONNIER ${ }^{1}$, AND Gerd WeIGELT ${ }^{2}$ \\ ${ }^{1}$ Department of Astronomy, University of Michigan, 918 Dennison Building, Ann Arbor, MI 48109-1090, USA \\ ${ }^{2}$ Max Planck Institut für Radioastronomie, Auf dem Hügel 69, 53121 Bonn, Germany \\ Received 2012 January 27; accepted 2012 April 5; published 2012 May 21
}

\begin{abstract}
V921 Scorpii is a close binary system (separation 0'025) showing the B[e]-phenomenon. The system is surrounded by an enigmatic bipolar nebula, which might have been shaped by episodic mass-loss events, possibly triggered by dynamical interactions between the companion and the circumprimary disk. In this paper, we investigate the spatial structure and kinematics of the circumprimary disk, with the aim to obtain new insights into the still strongly debated evolutionary stage. For this purpose, we combine, for the first time, infrared spectro-interferometry (VLTI/AMBER, $\lambda / \Delta \lambda=12,000$ ) and spectro-astrometry (VLT/CRIRES, $\lambda / \Delta \lambda=100,000$ ), which allows us to study the AU-scale distribution of circumstellar gas and dust with an unprecedented velocity resolution of $3 \mathrm{~km} \mathrm{~s}^{-1}$. Using a model-independent photocenter analysis technique, we find that the $\mathrm{Br} \gamma$-line-emitting gas rotates in the same plane as the dust disk. We can reproduce the wavelength-differential visibilities and phases and the double-peaked line profile using a Keplerian-rotating disk model. The derived mass of the central star is $5.4 \pm 0.4 M_{\odot} \cdot(d / 1150 \mathrm{pc})$, which is considerably lower than expected from the spectral classification, suggesting that V921 Sco might be more distant $(d \sim 2 \mathrm{kpc})$ than commonly assumed. Using the geometric information provided by our $\mathrm{Br} \gamma$ spectro-interferometric data and Paschen, Brackett, and Pfund line decrement measurements in 61 hydrogen recombination line transitions, we derive the density of the line-emitting gas $\left(N_{e}=(2-6) \times 10^{19} \mathrm{~m}^{-3}\right)$. Given that our measurements can be reproduced with a Keplerian velocity field without an outflowing velocity component and the non-detection of age-indicating spectroscopic diagnostics, our study provides new evidence for the pre-main-sequence nature of V921 Sco.
\end{abstract}

Key words: accretion, accretion disks - binaries: close - protoplanetary disks - stars: emission-line, $\mathrm{Be}$ - stars: individual (V921 Scorpii) - stars: pre-main sequence - techniques: interferometric

Online-only material: color figures

\section{INTRODUCTION}

The class of the $\mathrm{B}[\mathrm{e}]$ stars comprises some of the most peculiar and mysterious objects of stellar astrophysics. In particular, it has been found that the class-defining characteristics, which include infrared excess and strong permitted and forbidden line emission, are observed in a wide range of evolutionary stages, including stars of pre-main-sequence, post-main-sequence, and unknown nature (unclassified B[e] stars; Lamers et al. 1998). An enigmatic member of the latter category is V921 Scorpii (=Hen 3-1300, MWC 865, CD -42 11721). Despite more than one hundred studies that have been conducted over the last four decades, the stellar parameters and evolutionary stage of V921 Sco remain strongly debated. These uncertainties arise primarily from problems in distance estimation, where values between $160 \mathrm{pc}$ (Hillenbrand et al. 1992) and $2.6 \mathrm{kpc}$ (Shore et al. 1990) have been proposed. Furthermore, due to the lack of photospheric lines in the optical spectrum, the existing spectral classifications are based on rather indirect methods, such as photometric modeling or the ionization analysis of circumstellar emission lines. Accordingly, the derived effective temperatures (12,300-31,600 K; Hillenbrand et al. 1992; Cidale et al. 2001) and spectral types (Aep to B0[e]p; de Winter \& The 1990; Natta et al. 1993) exhibit a considerable spread, which prevents a clear Hertzsprung-Russell diagram classification and has lead authors to argue both for an evolved (supergiant;

\footnotetext{
* Based on observations made with ESO telescopes at the Paranal Observatory under the open-time program ID 084.C-0668(A, B) and with the Magellan Clay telescope.
}

Hutsemekers \& van Drom 1990; Borges Fernandes et al. 2007) and young (Herbig B[e]; de Winter \& The 1990; The et al. 1994; Benedettini et al. 1998; Habart et al. 2003; Acke et al. 2005; Acke \& van den Ancker 2006) object nature.

The aim of this paper is to gain new insights on the astrophysical nature of V921 Sco, and B[e] stars in general, by studying the spatial distribution and kinematics of the circumstellar gas and dust. These constraints will inform us about the diskformation mechanism (i.e., accretion versus excretion), and, thus, the evolutionary state of V921 Sco. For instance, accretion disks around young stars are believed to exhibit a Keplerian rotation profile, while the radiation-driven winds from evolved stars should exhibit a strong outflowing velocity component (Lamers \& Pauldrach 1991). Given the possible kiloparsec distance of V921 Sco, this task requires both high angular and high spectral resolution, which we achieve in our study by combining, for the first time, two highly complementary techniques, namely spectro-interferometry (VLTI/AMBER, providing milliarcsecond angular resolution and spectral resolution $R=12,000)$ and spectro-astrometry (VLT/CRIRES, $R=100,000$ ). From these spatially and spectrally resolved constraints, we derive the gas velocity field on scales down to a few stellar radii.

In a recent study (Kraus et al. 2012a, Paper I), we already obtained milliarcsecond-resolution continuum interferometric images of V921 Sco in three wavelength bands $(1.65,2.0$, and $2.3 \mu \mathrm{m}$ ) and discovered a close (separation $\rho \sim 25$ milliarcseconds (mas)) companion, as well as a continuum-emitting disk with an apparent size of $7.5 \pm 0.2$ mas (Gaussian FWHM along major axis). In addition, we obtained images of the surrounding 
large-scale bipolar nebula and detected multi-layered, shell-like substructures that might have been shaped by episodic massloss events. Based on roughly matching timescales between the estimated orbital period and the mass-ejection period, we suggested that the mass-ejection events might be triggered by the newly discovered companion.

For the stellar parameters of V921 Sco, we adopt in this study a luminosity $L_{\star}=(10 \pm 3) \times 10^{3} L_{\odot}$, effective temperature $T_{\text {eff }}=(14 \pm 1) \times 10^{3} \mathrm{~K}$, a distance of $d=1150 \pm 150 \mathrm{pc}$, $A_{V}=4.8 \pm 0.2$, and a stellar radius of $R_{\star}=17.3 \pm 0.6 R_{\odot}$ (Borges Fernandes et al. 2007). The implications of a modified set of fundamental parameters will be discussed in Section 5.2.

In the following, we present our spectro-interferometric (Section 2.1), spectro-astrometric (Section 2.2), and spectroscopic (Section 2.3) observations, followed by a discussion and quantitative modeling, both in the continuum (Section 3) and the $\mathrm{Br} \gamma$ line (Section 4). Finally, we will interpret our modeling results (Section 5) and conclude with a brief summary of our findings (Section 6).

\section{OBSERVATIONS}

\subsection{VLTI/AMBER Spectro-interferometry}

Infrared interferometers, such as ESO's Very Large Telescope Interferometer (VLTI), coherently combine the light from separate apertures in order to achieve an unprecedented angular resolution of a few milliarcseconds at infrared wavelengths. With a spectral resolving power of up to $R=\lambda / \Delta \lambda=12,000$, these instruments now enable investigations both in the continuum emission and in spatially and spectrally resolved gas-tracing lines. The measured visibility amplitudes, closure phases, and wavelength-differential phases (DPs) provide powerful constraints for model-fitting or can be used for the reconstruction of interferometric images.

First spectro-interferometric observations on V921 Sco using the VLTI near-infrared beam combiner instrument AMBER (Petrov et al. 2007) were presented by Kraus et al. (2008a), Kreplin et al. (2012), and in Paper I and provided low (LR-HK, $R=35)$ and medium (MR-K, $R=1500)$ spectral resolution. Here, we present new observations using AMBER's unique high spectral resolution mode (HR-K, $R=12,000)$. The new data sets were obtained on 2010 March 3 and 2010 August 26 using the $8.2 \mathrm{~m}$ unit telescopes UT2, UT3, and UT4 and cover a wavelength window around the $\mathrm{Br} \gamma 2.16602 \mu \mathrm{m}$ line.

In order to yield sufficient signal-to-noise ratio $(\mathrm{S} / \mathrm{N})$ for our high spectral resolution observations, we employed the FINITO fringe tracker instrument (Gai et al. 2004; Le Bouquin et al. 2008). This instrument measures and corrects the atmosphereinduced phase perturbations and allowed us to record the optical path delay stabilized interferograms with a long detector integration time of $3 \mathrm{~s}$. Unfortunately, the tracking performance of FINITO was degraded for the longest baselines due to the low visibility contrast in this baseline regime. Based on the poor $\mathrm{S} / \mathrm{N}$, we rejected the visibility measurements for one baseline from 2010 March 3 (UT2-UT4) and two baselines from 2010 August 26 (UT2-UT4, UT3-UT4). The remaining baselines exhibit fringe $\mathrm{S} / \mathrm{Ns}$ up to $3.5,4.5$, and 14 , and cover baseline lengths between 36.7 and $84.3 \mathrm{~m}$ and PAs between $22^{\circ}$ and $292^{\circ}$ (Figure 1). The data show a double-peaked, rising visibility profile in the $\mathrm{Br} \gamma$ line as well as non-zero DPs (Figure 2).

The wavelength calibration was done using atmospheric telluric features close to the $\mathrm{Br} \gamma$ line and by applying a heliocentric-barycentric system correction with heliocentric

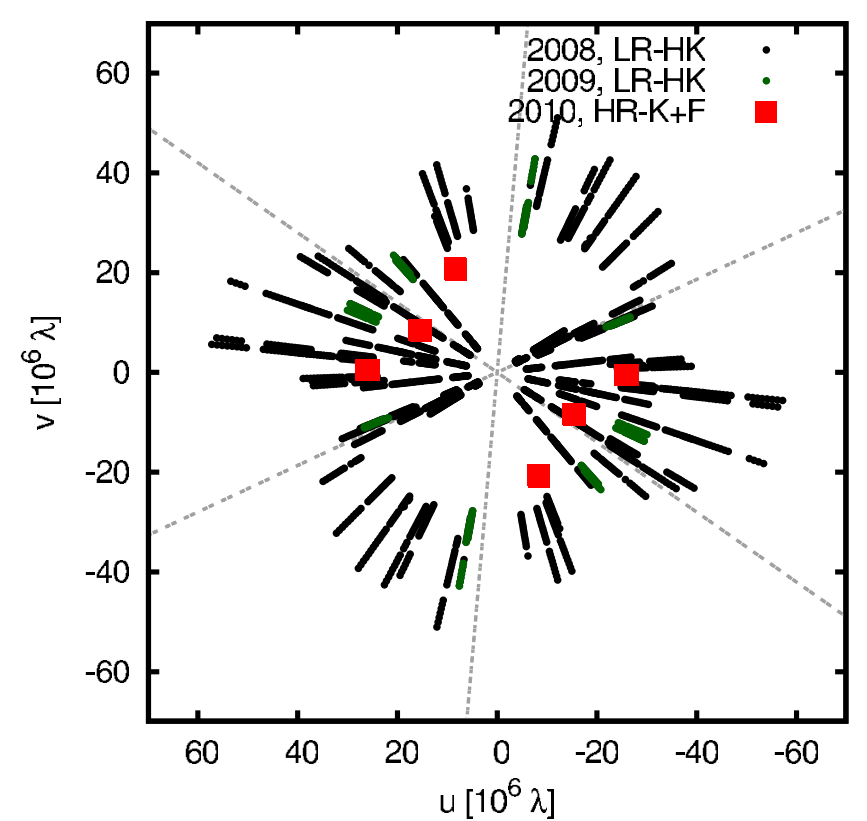

Figure 1. $u v$-coverage of our VLTI/AMBER observations using the LR-HK mode (black dots, Paper I) and HR-K mode (red squared, this paper), as well as the slit orientation of the spectro-astrometric observations (dashed gray lines).

(A color version of this figure is available in the online journal.)

velocities of $+28.33 \mathrm{~km} \mathrm{~s}^{-1}$ (2010 March 3) and $-27.09 \mathrm{~km}^{-1}$ (2010 August 26), respectively.

Each observation on V921 Sco was accompanied by observations on the interferometric calibrator HD 161068, for which we assume a uniform-disk diameter of $1.448 \pm 0.019$ mas (Mérand et al. 2006). Both for the science and calibrator star observations, we extract raw visibilities, closure phases, and DPs using the amdlib (V3.0) data reduction software (Tatulli et al. 2007b; Chelli et al. 2009).

Due to the influence of residual vibration, it is known that AMBER+FINITO observations with long DIT, such as used for our HR-K mode observations, might result in a poor absolute visibility calibration, while the wavelength-differential observables are only marginally affected (e.g., Tatulli et al. 2007a). To recalibrate the absolute continuum visibility level of the HR-K observations, we make use of our earlier LR-HK observations (Paper I) and the best-fit continuum model (Model DISK), which will be described in more detail in Section 3. We investigated the influence of frame selection in the course of our data reduction procedure and find that the wavelengthdifferential signatures are very robust when selecting, for instance, the $80 \%, 50 \%, 20 \%$, or $10 \%$ of frames with the best $\mathrm{S} / \mathrm{N}$. Given that the statistical noise increases with decreasing frame number and that low-S/N frames are downweighted during the averaging process in a natural fashion, we decided to employ the observables from the full, unselected data sets for our analysis.

In order to associate the closure phase sign with the onsky orientation, we use a reference data $\operatorname{set}^{3}$ on the binary star $\theta^{1}$ Orionis C (Kraus et al. 2009b). To calibrate the wavelengthdifferential phases, we make use of the fact that the continuum photocenter is systematically offset with respect to the $\mathrm{Br} \gamma$-line emission due to the presence of a close companion star. Using the well-established continuum closure phase sign calibration,

\footnotetext{
3 The reference data set can be accessed on the Web site: http://www.stefan-kraus.com/files/amber.htm.
} 


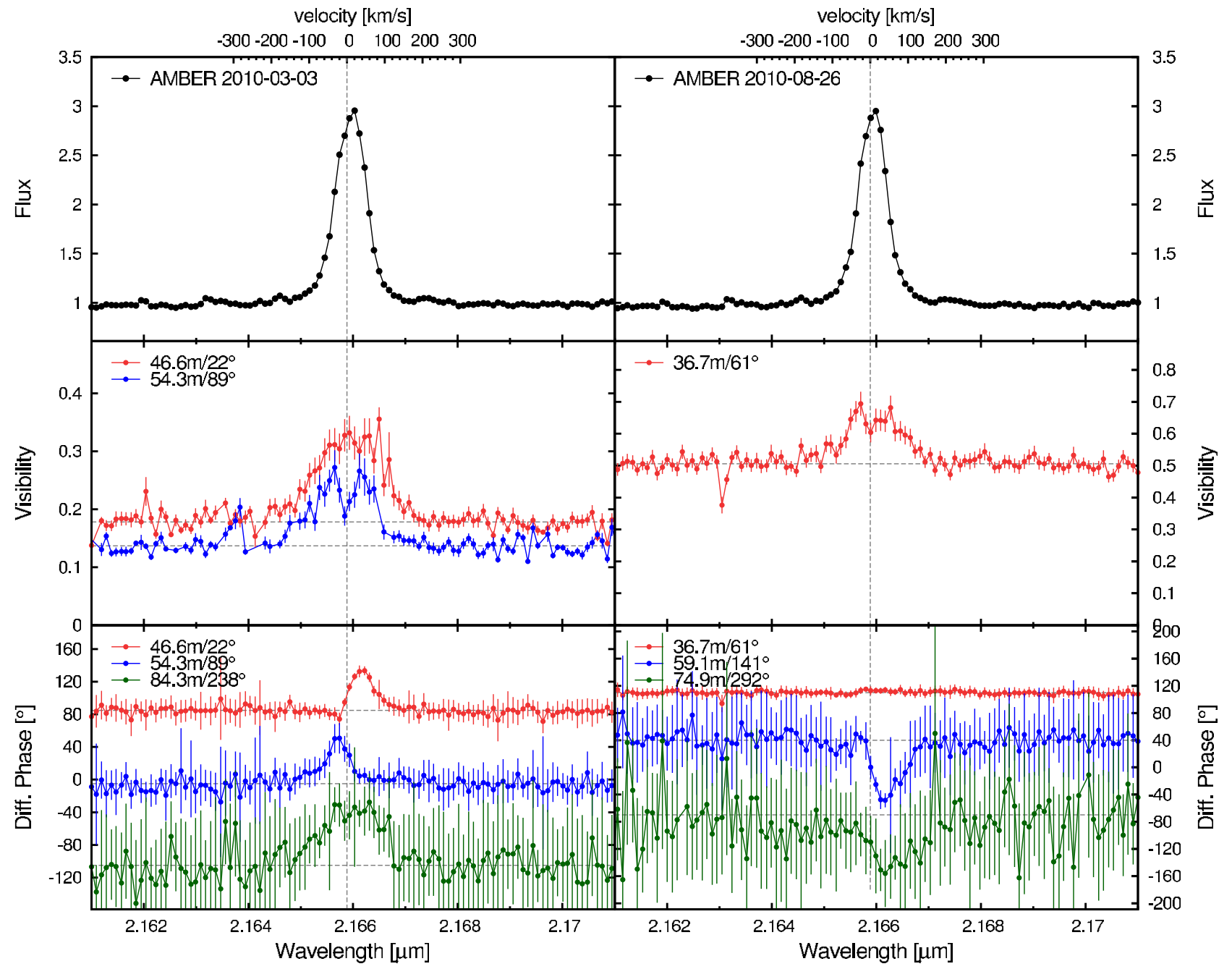

Figure 2. Spectra (top), visibilities (middle), and DPs (bottom) derived from our VLTI/AMBER observations with spectral resolution $R=12,000$ obtained on 2010 March 3 (left) and 2010 August 26 (right). For the DP measurements, we add an arbitrary offset (dashed line) for clarity.

(A color version of this figure is available in the online journal.)

we first determine the position angle ${ }^{4}$ (P.A.) of the companion star (Section 3) and then adjust the DP sign in our spectrointerferometric and spectro-astrometric data in order to match the direction of the continuum photocenter displacement. This procedure calibrates the DP sign unambiguously and might serve also as a reference for other studies using AMBER's HR mode. Recently, we employed this calibration in order to determine the rotation sense of the disk around the classical Be star $\beta$ CMi (Kraus et al. 2012b). Since $\beta$ CMi was observed using both AMBER's MR and HR mode, we can also extend the calibration to AMBER MR-mode observations, which resulted in a recalibration of the $\zeta$ Tau rotation sense (Kraus et al. 2012b) compared with earlier studies (Štefl et al. 2009).

The statistical error bars on the DPs vary strongly for the different baselines, which is both a result of the low fringe contrast at the longest baselines and of differences in the vibration properties of the UTs. These differences might give overproportional weight to the short baselines in our $\chi^{2}$-fitting procedure. Therefore, based on the typical root-mean-square noise in the continuum channels, we include a minimum DP error of $20^{\circ}$ for all baselines in the fitting procedure.

\footnotetext{
4 In this paper, all position angles are measured east of north.
}

\subsection{VLT/CRIRES Spectro-astrometry}

Spectro-astrometry uses high $\mathrm{S} / \mathrm{N}$ long-slit spectra to measure the centroid position of an unresolved object as a function of wavelength. Since the centroid position can be measured with much higher precision than the size of the point-spread function (Bailey 1998b), this method allows one to measure sub-mas photocenter displacements in spectrally resolved emission lines. While the earliest astronomical applications in the field of star formation focused on the detection of close companions (e.g., Bailey 1998a), later studies also successfully applied spectroastrometry to more general cases, such as outflow signatures (Takami et al. 2003; Whelan et al. 2005) or the characterization of the gas kinematics in protoplanetary disks (Pontoppidan et al. 2011; Goto et al. 2012).

Our spectro-astrometric observations on V921 Sco were obtained using the VLT near-infrared high-resolution spectrograph CRIRES (Kaeufl et al. 2004). The measurements were obtained on 2010 March 4, UT 07:51 to 08:58 using an integration time of $5 \mathrm{~s}$ and a slit width of $0^{\prime} \cdot 2$, resulting in a spectral resolution of $R=100,000$. In order to apply the spectro-astrometry technique, we recorded spectra toward three different P.A.s $\left(55^{\circ}\right.$, $\left.115^{\circ}, 175^{\circ}\right)$, and the corresponding anti-parallel P.A.s $\left(235^{\circ}\right.$, $295^{\circ}, 355^{\circ}$ ), complementing the P.A.s probed by interferometry 

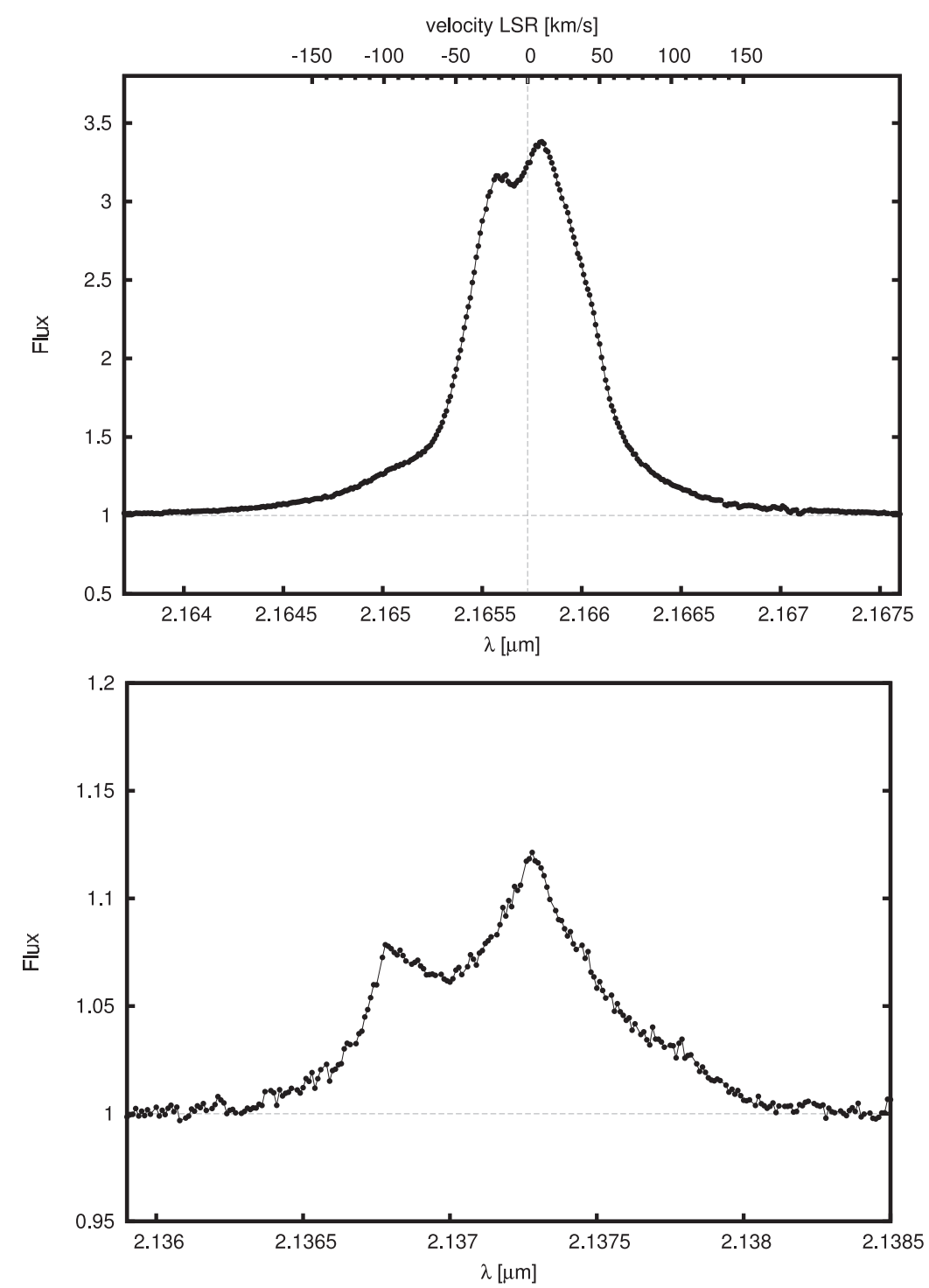

Figure 3. VLT/CRIRES $(R=100,000)$ spectra obtained on V921 Sco in the $\mathrm{Br} \gamma$ and $\mathrm{Mg}$ II line.

(Figure 1). To maximize the flux in the slit, the spectra were recorded using the STRAP adaptive optics system, resulting in a typical PSF Gaussian FWHM of $\sim 115$ mas. The measured spectra were corrected to the heliocentric-barycentric system using a heliocentric velocity of $+28.36 \mathrm{~km} \mathrm{~s}^{-1}$. The spectra were extracted using the ESO CRIRES data reduction pipeline (version 1.12.0).

Besides the $\operatorname{Br} \gamma 2.166078 \mu \mathrm{m}$ line, which was covered by CRIRES detector 3, our observation also covered the $\mathrm{Mg}$ II $2.137 \mu \mathrm{m}$ line in detector 1 . This line has also been detected in other B[e] stars (Clark et al. 1999). To compute velocities in the local standard of rest, we assumed a systemic velocity of $v_{0}=-20 \mathrm{~km} \mathrm{~s}^{-1}$, which we adopt from the forbidden line measurements by Borges Fernandes et al. (2007).

In order to correct the spectrum for telluric spectral features, we observed the early G-type star Hip84425 and we modeled the intrinsic $\mathrm{Br} \gamma$ absorption using an infrared solar spectrum recorded with ACE-FTS (Hase et al. 2010).

The final spectra are shown in Figure 3 and reveal a very narrow (FWHM $0.0006560 \mu \mathrm{m}$ or $\approx 91 \mathrm{~km} \mathrm{~s}^{-1}$ ) and doublepeaked line profile with a very small peak separation of just $\sim 0.000184 \mu \mathrm{m}\left(\sim 25 \mathrm{~km} \mathrm{~s}^{-1}\right)$. In addition to the $\mathrm{Br} \gamma$-line spectrum, which will be discussed and modeled in Section 4, we have also recorded the $\mathrm{Mg}$ II $2.137 \mu \mathrm{m}$ line. For the $\mathrm{Mg}$ II line, the peak separation is significantly wider $(\sim 0.000500 \mu \mathrm{m}$ or $70 \mathrm{~km} \mathrm{~s}^{-1}$ ), indicating that this line originates at smaller stellocentric radii in the circumstellar environment. This finding is consistent with our non-detection of a spectro-astrometric signal within this line.

To derive the astrometric signal, we compute for each spectral channel $v$ the beam centroid position (Pontoppidan et al. 2011) in the spatial direction

$$
X^{p, a}(v)=K \frac{\sum_{i} x_{i}(v) F_{i}(v)}{\sum_{i} F_{i}(v)},
$$

where $i$ defines the size of the virtual aperture (we use \pm 1 pixel around the center of the PSF) and $K$ is defined as the ratio between the flux in the virtual aperture and the total $\operatorname{PSF}(\sim 1.5)$. The astrometric signals are derived for each P.A. $\left(X^{p}\right)$ and its anti-parallel counterpart $\left(X^{a}\right)$ and then subtracted in order to remove potential artifacts (Brannigan et al. 2006):

$$
X(v)=\left(X^{p}(v)-X^{a}(v)\right) / 2 .
$$

The interpretation of spectro-astrometric signals is complicated by the fact that this technique measures only the first-order 
momentum in the brightness distribution (relative position), but is insensitive to higher-order momenta (angular size, asymmetry, kurtosis; see Lachaume 2003) as well as the continuumemitting geometry, which limits this technique to relatively simple cases or requires additional model assumptions. In this study, we make use of the fact that photocenter displacements measured with spectro-astrometry are mathematically equivalent to the DPs measured in spectro-interferometry, which allows us to directly combine the spectro-interferometric (visibility, DP, closure phases) and spectro-astrometric observables (photocenter displacements) for quantitative modeling (Section 4.2), providing unique constraints on the AU-scale spatial distribution and kinematics of the circumstellar gas and dust. For this purpose, we translate the measured astrometric signal into the equivalent DP using the relation

$$
\phi=-\frac{2 \pi X(v)}{\sigma},
$$

where $\sigma$ is the FWHM of the PSF measured in the spectrum (typically $\sim 150$ mas). In order to give a similar weight to the AMBER and CRIRES measurements, we assume a minimum DP error of $1^{\circ}$ for our CRIRES DPs.

\subsection{Magellan/FIRE Near-infrared Spectroscopy}

We recorded a high-resolution, high-S/N near-infrared spectrum of V921 Sco using the FIRE Echelle spectrograph (Simcoe et al. 2008) mounted at the Magellan/Baade $6.5 \mathrm{~m}$ telescope. With a 0.45 slit, this instrument provides a spectral resolution $R=8000$ and a wide wavelength coverage from 0.8 to $2.5 \mu \mathrm{m}$. The spectrum was recorded on 2011 March 12 using an A-B-B-A dithering pattern with DITs of $10 \mathrm{~s}$ (high gain mode, to obtain high $\mathrm{S} / \mathrm{N}$ in the $z$ and $J$ band) and $5 \mathrm{~s}$ (low gain mode, in order to avoid saturation in the $H$ and $K$ bands). The slit was oriented along the disk polar axis derived from our interferometric observations (P.A. $=56^{\circ}$ ). To correct for telluric features, we observed the A0V-type standard star HD 122945. The data reduction was performed using the standard FIRE data reduction pipeline developed at MIT, which performs also a spectro-photometric calibration based using the photometry of the standard star. Trying different combinations of target star/standard star data sets, we find that the derived spectral slopes are consistent on the level of a few percent, while the derived absolute flux levels show some larger scatter in the $\sim 5 \%-10 \%$ range. In order to improve on the calibration, we fit a fifth-order polynomial in order to extract the spectral slope and then use archival ISO photometry in order to recalibrate the absolute flux at $2.4 \mu \mathrm{m}$ which yields also a satisfying match with 2 MASS $J-, H$-, and $K$-band photometry (the derived flux densities match within 20\%). The derived absolute-calibrated FIRE spectrum is corrected for reddening $\left(A_{V}=4.8 \pm 0.2\right.$; Borges Fernandes et al. 2007) using the extinction law from Cardelli et al. (1989, $\left.R_{V}=3.1\right)$.

At the longest wavelengths, the $\mathrm{S} / \mathrm{N}$ in our final spectrum is reduced compared with the $J$ and $H$ bands since some of the recorded frames had to be rejected due to saturation in this part of the spectrum. In particular, the saturation occurs in sky lines, resulting in some narrow spikes in the final corrected spectrum.

\section{RESULTS: CONTINUUM GEOMETRY}

In the following, we investigate the continuum structure of the circumprimary disks using the VLTI/AMBER low spectral dispersion data presented in Paper I. In this earlier study, the circumprimary disk emission was approximated with a Gaussian brightness distribution (in the following denoted by "GAUSS" model), which provided a sufficient representation to extract the astrometric information for the companion star. However, the relatively large $\chi_{r}^{2}$-value of 4.30 already indicates that this simple geometry does not provide an appropriate representation of the circumprimary disk structure. Therefore, we consider here a more realistic parameterization for the structure of the circumprimary disk, using a disk temperature-gradient model (in the following denoted by "DISK" model).

In the DISK model, the emission extends from an inner truncation radius $R_{\text {in }}$ to an outer radius $R_{\text {out }}$ and radiates as a blackbody with $T(r)=T_{\text {in }}\left(r / R_{\text {in }}\right)^{-q}$, where $T_{\text {in }}$ denotes the temperature at the inner disk radius and $q$ is the temperature power-law index. In addition, we introduce the inclination angle $i$ and P.A. $\varphi$, which define the projection ratio $\left(\cos i=R_{\text {minor }} / R_{\text {major }}\right)$ and on-sky orientation of the disk major axis. The inclination is measured from the polar axis (i.e., $i=0^{\circ}$ is pole-on). As in our earlier modeling attempts, the DISK model includes the photospheric emission of the primary star and secondary star, which are parameterized by the companion separation $(\rho)$ and P.A. $(\Theta)$, the angular extension of the circumsecondary material (given by a Gaussian with FWHM $\theta_{B}$ ), and two parameters $\left(F_{\mathrm{B}} / F_{\text {tot }}(2 \mu \mathrm{m}), s\right)$ to describe the photospheric flux contributions of V921 Sco B to the total flux as a function of wavelength: $F_{\mathrm{B}} / F_{\text {tot }}(\lambda)=F_{\mathrm{B}} / F_{\text {tot }}(2 \mu \mathrm{m})+s \cdot(\lambda-2 \mu \mathrm{m})$.

We employ a Levenberg-Marquardt least-square fitting procedure and find the best-fit solution by minimizing the likelihood estimator $\chi_{r}^{2}=\chi_{r, V}^{2}+\chi_{r, \Phi}^{2}$, where $\chi_{r, V}^{2}$ and $\chi_{r, \Phi}^{2}$ are the reduced least square between the measured and model visibilities and closure phases, respectively. The parameter uncertainties have been estimated using the bootstrapping technique.

Given that the absolute calibration of the AMBER+FINITO HR observations is not very reliable due to the use of long integration times and a potential residual phase jitter, we decided to recalibrate the absolute visibility level using the detailed continuum model discussed in Section 3 (Model DISK). Since the HR data were recorded in 2010, we extrapolated the orbital motion of the companion to this later epoch ( $\rho=25.41$ mas, $\Theta=339^{\circ}$ ), but note that the continuum geometry has only a marginal effect on our model-fitting results in the strong $\mathrm{Br} \gamma$ line of V921 Sco.

The DISK model provides a significant improvement compared to the GAUSS model, which also reflects in an improved $\chi_{r}^{2}$-values of 1.86 (versus 4.88) for the 2008 data and 3.02 (versus 3.39) for the 2009 data, respectively. In contrast to the GAUSS model, the DISK model also provides a physically motivated parameterization for the wavelength-dependent changes in the source geometry, allowing us to fit all wavelength channels simultaneously. The resulting best-fit parameters for the various models are listed in Table 1. We plot in Figure 4 the measured observables versus the prediction of the DISK model. Besides the spatially resolved emission around the primary component, we also checked whether the fit results can be improved by including spatially extended emission around the northern (secondary) component, but were not able to find a significant improvement.

\section{RESULTS: $\operatorname{Br} \gamma$-LINE GEOMETRY}

\subsection{Two-dimensional Photocenter Analysis}

For a first, qualitative interpretation (Section 4.1) of the gas kinematics, we derive the photocenter displacement of the 


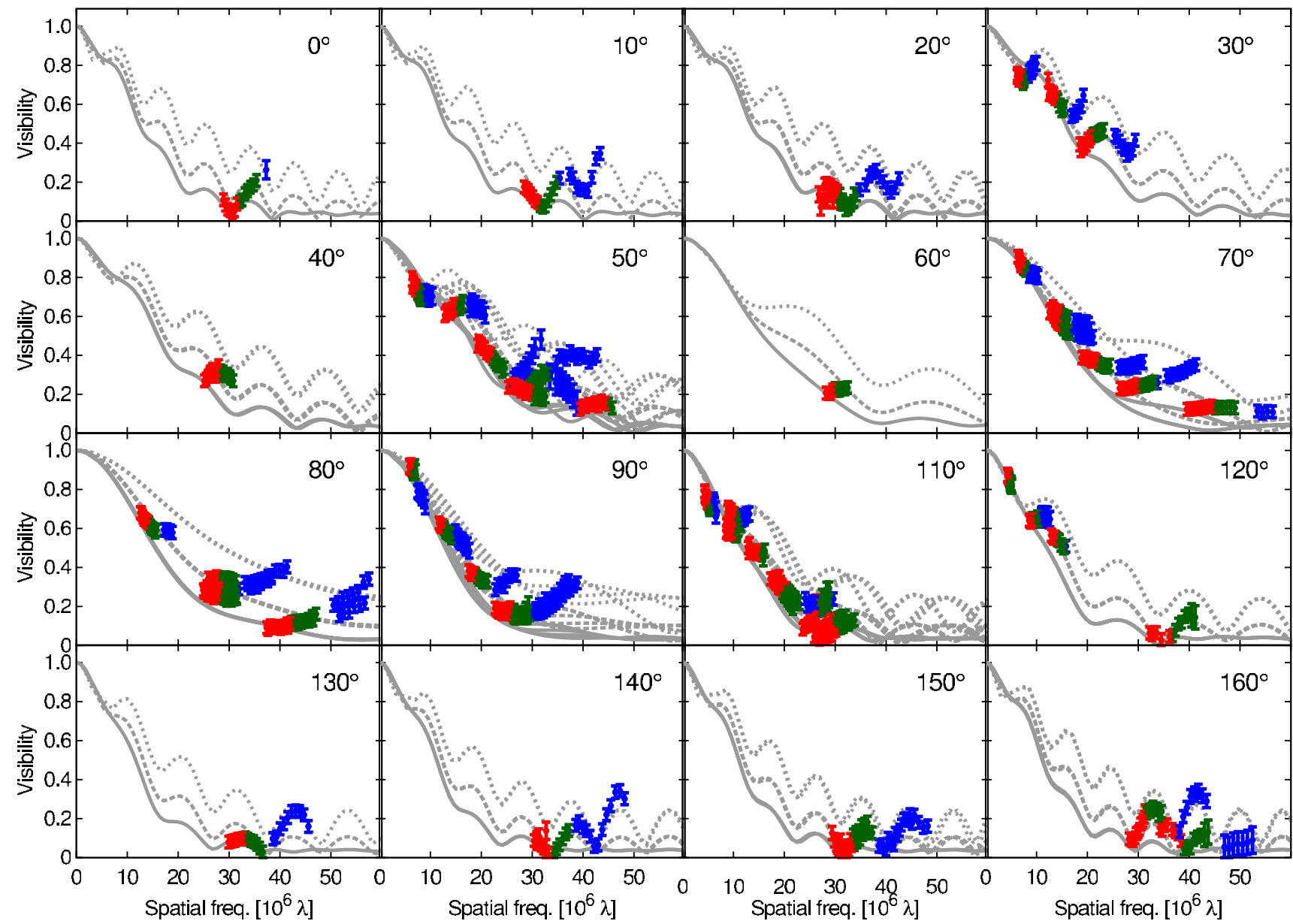

Figure 4. VLTI/AMBER visibilities (LR-HK mode) of V921 Sco in the $H$ and $K$ bands, plotted as a function of spatial frequency. The different panels represent position angle bins, covering $10^{\circ}$ each. The gray model curves correspond to our best-fit temperature gradient disk model (DISK) and were computed for the lower (1.45 $\mu \mathrm{m}$, dotted line), central ( $2 \mu \mathrm{m}$, dashed line), and upper $(2.55 \mu \mathrm{m}$, solid line) part of the wavelength range. For clarity, the data points are color-coded according to the wavelength of the corresponding spectral channel (blue: $1.4 \mu \mathrm{m} \leqslant \lambda<1.9 \mu \mathrm{m}$; green: $1.9 \mu \mathrm{m} \leqslant \lambda<2.15 \mu \mathrm{m}$; red: $2.15 \mu \mathrm{m} \leqslant \lambda<2.5 \mu \mathrm{m}$ ).

(A color version of this figure is available in the online journal.)

Table 1

Model-fitting Results for the VLTI/AMBER Continuum Observations (Section 3)

\begin{tabular}{|c|c|c|c|c|c|c|c|c|c|c|c|c|c|c|c|}
\hline \multirow[t]{2}{*}{ Epoch } & \multirow[t]{2}{*}{ Model } & \multicolumn{5}{|c|}{ Secondary Star } & \multicolumn{6}{|c|}{ Circumprimary Disk } & \multirow[b]{2}{*}{$\chi_{\mathrm{r}, \mathrm{V}}^{2}$} & \multirow[b]{2}{*}{$\chi_{\mathrm{r}, \Phi}^{2}$} & \multirow[b]{2}{*}{$\chi_{\mathrm{r}}^{2}$} \\
\hline & & $\begin{array}{c}\Theta \\
\left(^{\circ}\right)\end{array}$ & $\begin{array}{c}\rho \\
(\mathrm{mas})\end{array}$ & $\begin{array}{c}\theta_{\mathrm{B}} \\
(\mathrm{mas})\end{array}$ & $\begin{array}{c}\frac{F_{\mathrm{B}}}{F_{\mathrm{tot}}} \\
\text { (at } 2 \mu \mathrm{m})\end{array}$ & $\begin{array}{c}s \\
\left(\mu \mathrm{m}^{-1}\right)\end{array}$ & $\begin{array}{c}\theta_{\mathrm{A}} \\
(\mathrm{mas})\end{array}$ & $\begin{array}{c}i \\
\left(^{\circ}\right)\end{array}$ & $\begin{array}{c}\varphi \\
\left({ }^{\circ}\right)\end{array}$ & $\begin{array}{c}R_{\text {in }} \\
\text { (mas) }\end{array}$ & $\begin{array}{c}R_{\text {out }} \\
\text { (mas) }\end{array}$ & $q$ & & & \\
\hline 2008 & GAUSS & $\begin{array}{r}353.8 \\
\pm 1.6\end{array}$ & $\begin{array}{r}25.0 \\
\pm 0.8\end{array}$ & $\leqslant 0.2$ & $\begin{array}{r}0.054 \\
\pm 0.018\end{array}$ & $\begin{array}{l}-0.0056 \\
\pm 0.019\end{array}$ & $\begin{array}{r}7.5 \\
\pm 0.2\end{array}$ & $\begin{array}{r}50.3 \\
\pm 1.9\end{array}$ & $\begin{array}{l}147.8 \\
\pm 4.3\end{array}$ & $\ldots$ & $\ldots$ & $\ldots$ & 4.30 & 3.09 & 4.88 \\
\hline 2008 & DISK & $\begin{array}{r}354.0 \\
\pm 1.0 \\
\end{array}$ & $\begin{array}{r}24.9 \\
\pm 0.5 \\
\end{array}$ & $\leqslant 0.7$ & $\begin{array}{l}0.066 \\
\pm 0.04 \\
\end{array}$ & $\begin{array}{l}-0.051 \\
\pm 0.01 \\
\end{array}$ & $\ldots$ & $\begin{array}{l}48.8 \\
\pm 4 \\
\end{array}$ & $\begin{array}{l}145.0 \\
\pm 8.4 \\
\end{array}$ & $\begin{array}{c}1.59 \\
\pm 0.25 \\
\end{array}$ & $>9.7$ & $\begin{array}{c}0.36 \\
\pm 0.08 \\
\end{array}$ & 1.86 & 1.86 & 1.86 \\
\hline 2009 & GAUSS & $\begin{array}{r}347.3 \\
\pm 1.0\end{array}$ & $\begin{array}{r}25.5 \\
\pm 1.2\end{array}$ & $\leqslant 0.2^{\mathrm{a}}$ & $0.054^{\mathrm{a}}$ & $-0.0056^{\mathrm{a}}$ & $7.5^{\mathrm{a}}$ & $50.3^{\mathrm{a}}$ & $147.8^{\mathrm{a}}$ & $\ldots$ & $\ldots$ & $\ldots$ & 4.17 & 1.78 & 3.39 \\
\hline 2009 & DISK & $\begin{array}{r}347.4 \\
\pm 1.1\end{array}$ & $\begin{array}{r}25.4 \\
\pm 0.7\end{array}$ & $0.7^{\mathrm{a}}$ & $\begin{array}{r}0.086 \\
\pm 0.012\end{array}$ & $-0.051^{\mathrm{a}}$ & $\ldots$ & $48.8^{\mathrm{a}}$ & $145.0^{\mathrm{a}}$ & $1.59^{\mathrm{a}}$ & $9.7^{\mathrm{a}}$ & $0.36^{\mathrm{a}}$ & 3.26 & 2.52 & 3.02 \\
\hline
\end{tabular}

Note. ${ }^{a}$ In our fitting procedure, this parameter was kept fixed.

line-emitting region with respect to the continuum emission using our CRIRES spectro-astrometric data. For this purpose, it is necessary to separate the line spectro-astrometric signal from the underlying continuum contributions. To determine the continuum level, we fit a high-order polynomial function $X_{c}$ to the astrometric signal derived from the continuum channels. This function is then subtracted from the astrometric signal in the line channels and weighted by the continuum-to-line flux ratio $F_{c} / F_{l}$ :

$$
X_{l}(v)=\left(X(v)-X_{c}(v)\right)\left(1+F_{c}(v) / F_{l}(v)\right) .
$$

The derived photocenter of the line velocity channels is significantly offset with respect to each other (clearly indicating the gas kinematics) and also shows a displacement with respect to the photocenter of the continuum channels (Figure 5). This 

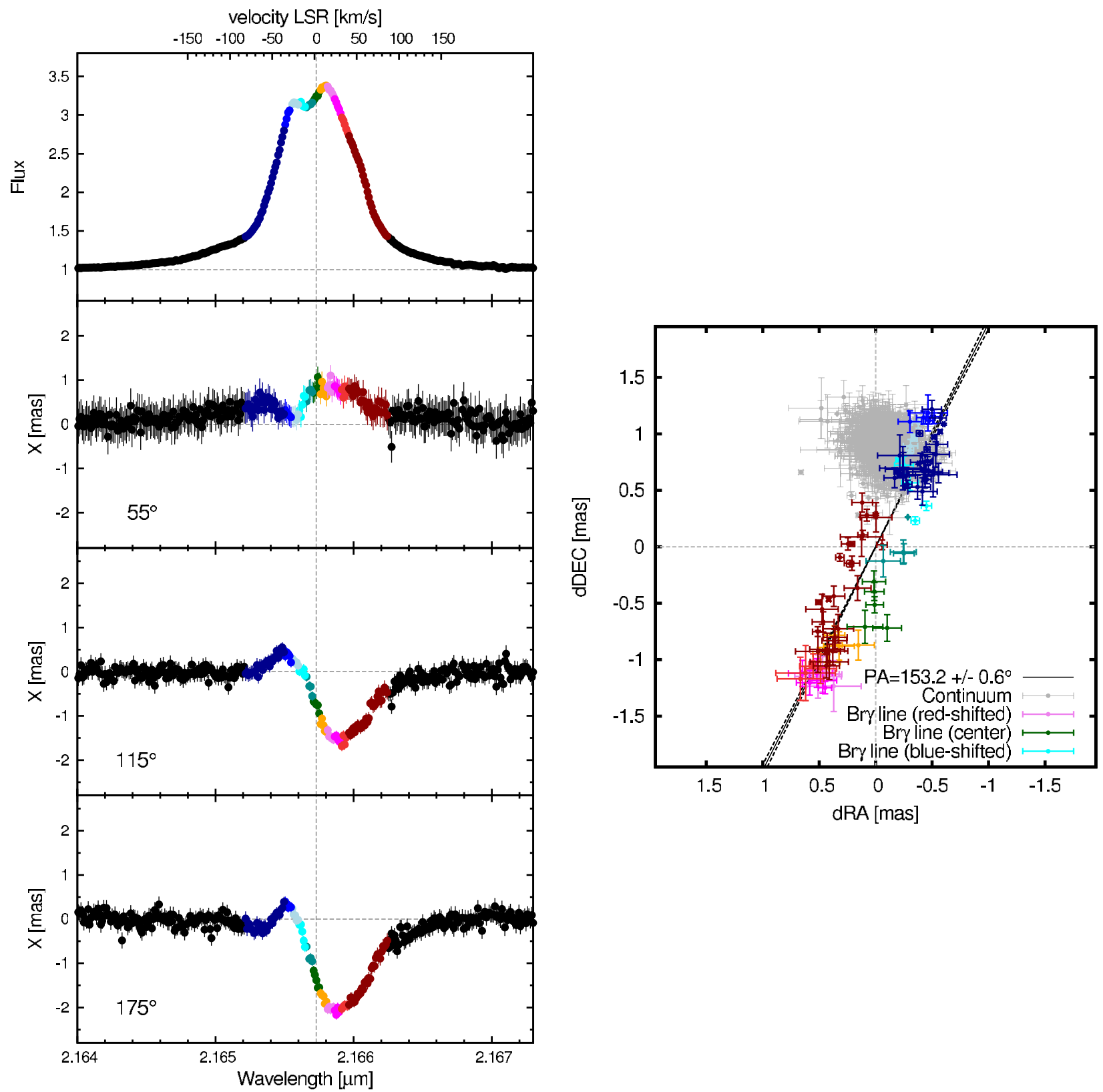

Figure 5. Left: spectrum (top) and spectro-astrometric signal (second to fourth panels from top) derived from our VLT/CRIRES $(R=100,000)$ observations. Right: derived two-dimensional photocenter displacements, where the different spectral channels are represented by different colors, matching the color coding in the left panel. From the photocenter displacements measured in the different spectral channels, we derive the disk rotation plane to $153: 2 \pm 0.6$. The continuum spectral channels (gray points) are offset with respect to the center of light of the line emission (origin of the coordinate system) due to the presence of the companion.

(A color version of this figure is available in the online journal.)

displacement reflects the fact that the center of gravity of the continuum emission does not coincide with the location of the primary star, but is displaced toward the position of the companion. We calculate the center of gravity both for the line channels and the continuum channels and compute their relative displacement vector $\boldsymbol{v}_{c-l}$, which is related to the companion separation $\rho$, P.A. $\Theta$, and the flux ratio $F_{\mathrm{B}} / F_{\text {tot }}$ by

$$
\boldsymbol{v}_{c-l}=\rho \frac{F_{\mathrm{B}}}{F_{\mathrm{tot}}}\left(\begin{array}{c}
\sin \Theta \\
\cos \Theta
\end{array}\right) .
$$

Based on our continuum model fits from 2008 and 2009 (Table 1), we fix $\rho=25.13$ mas and then determine the companion P.A. $\Theta$ and the flux ratio $F_{B} / F_{\text {tot }}$ from the CRIRES observations. Applying this procedure yields $\Theta=353^{\circ} \pm$ $9^{\circ}$ (which is in reasonable agreement with the astrometry determined with AMBER; Table 1) and $F_{B} / F_{\text {tot }}=0.053$, which is lower than the flux ratio determined with interferometry for epochs 2008 and 2009, possibly indicating variability.

The photocenters in the blue- and redshifted line wings are displaced in the opposite direction with respect to each other. Also, the highest gas velocities (dark red points and dark blue points in Figure 5) emerge from smaller stellocentric distances than intermediate velocities, which suggests a rotation-dominated velocity profile. From the distribution of the individual photocenter offsets, we derive the disk plane orientation to $153.2 \pm 0.6$.

In contrast to CRIRES, AMBER is able to spatially resolve the geometry of the line-emitting region, entering a regime where higher-order geometric effects are probed. Therefore, 

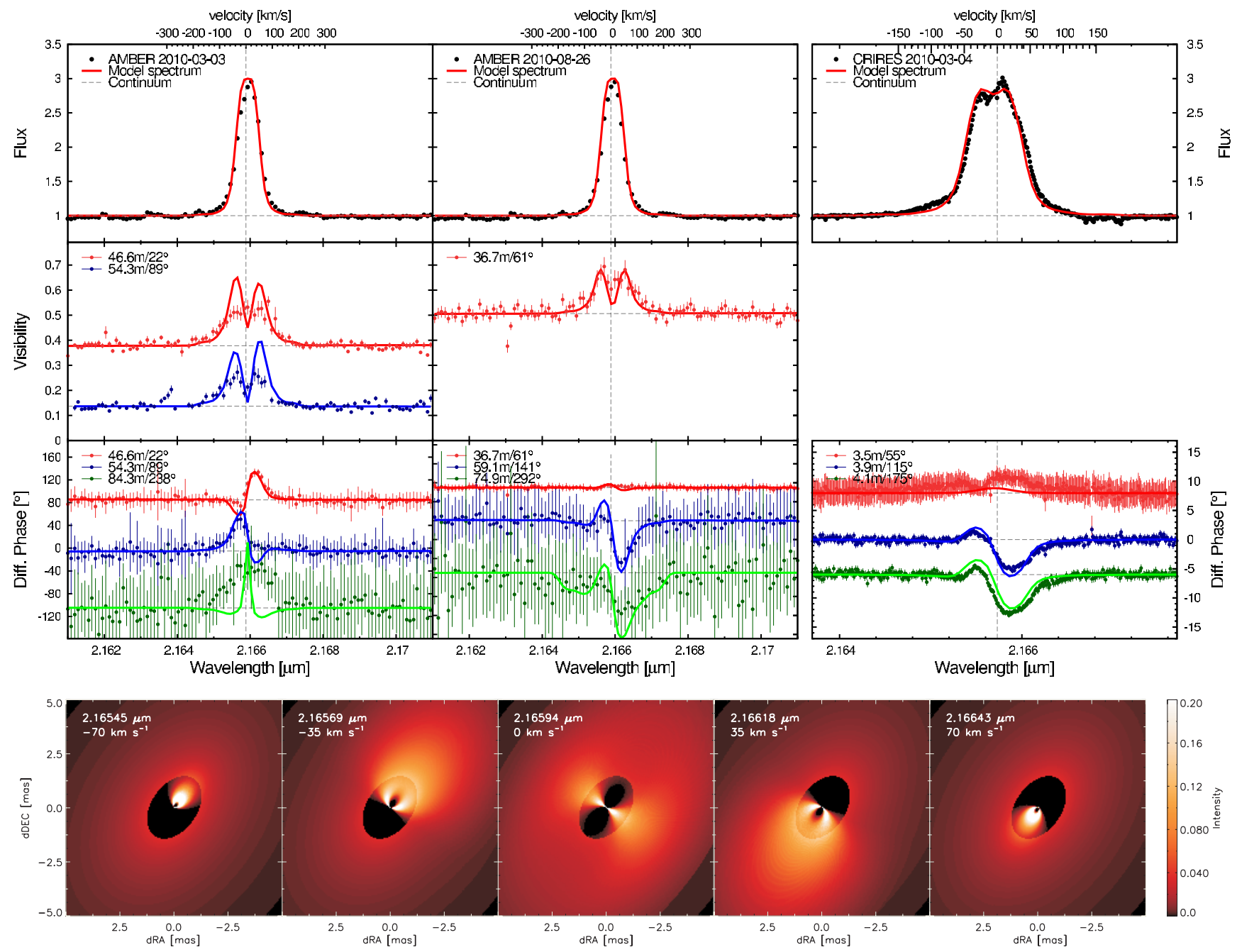

Figure 6. Top: spectra (first row), visibilities (second row), and differential phases (third row) derived from our AMBER and CRIRES Br $\gamma$ observations, overplotted with the Keplerian disk model (Section 4.2). For each interferometric baseline, we give the corresponding projected baseline length and P.A. in the label. In order to make the visibility profiles in the left panel clearly distinguishable, we added an offset of 0.2 to the visibility corresponding to the baseline $46.6 \mathrm{~m} / 22^{\circ}$. Bottom: to illustrate our kinematical model we show channel maps for some representative wavelengths, including the $\mathrm{Br} \gamma$ line and the continuum emission. The continuumemitting disk is truncated at a distance of 1.59 mas, while the Br $\gamma$-emitting material extends inward to a few stellar radius ( $\lesssim 0.5$ mas). The companion star V921 Sco $\mathrm{B}$ is also included in these simulations, but located outside of the FOV in these model images.

(A color version of this figure is available in the online journal.)

in the following two sections, we will employ a quantitative modeling in order to interpret the combined spectro-astrometric and spectro-interferometric observations.

\subsection{Model Fitting: Keplerian Disk}

Given the indications for a rotation-dominated velocity field provided by our model-independent photocenter analysis (Section 4.1), we first test whether a Keplerian velocity field might reproduce our spectroscopic, spectro-astrometric, and spectro-interferometric data quantitatively.

For this purpose, we employ a Keplerian disk model $(v(r)=$ $\sqrt{G M_{\star} / r}$ ), which we have already successfully applied to AMBER high spectral dispersion data on the classical Be star $\beta$ CMi (Kraus et al. 2012b). The model assumes that the lineemitting gas is optically thin and located in a geometrically thin disk, where the radial intensity profile is parameterized with a power law $\left(I_{l}(r) \propto r^{q}\right)$. The gas emission extends from an inner truncation radius $\left(R_{\text {in }}\right)$ to an outer truncation radius $\left(R_{\text {out }}\right)$, which we parameterize with a Fermi-type function in order to avoid artificial edges (see Equation (1) in Kraus et al. 2008b, where the width of the truncation region $\epsilon$ was chosen to 0.1 ). In our study on $\beta$ CMi (Kraus et al. 2012b), we investigated the DP signatures induced by photospheric absorption and found that even for a relatively extended stellar surface (equatorial radius of $0.36 \mathrm{mas}$ ), the induced DPs are $<0.5$. For V921 Sco, the influence of photospheric absorption is likely $\sim 50$ times smaller due to the smaller apparent stellar radius $(\sim 0.07$ mas $)$ and the significantly larger equivalent width of the $\mathrm{Br} \gamma$ emission line. Therefore, these signatures are about four orders of magnitude smaller than the disk kinematical signatures and can be safely neglected in our modeling process.

As discussed in Section 4.1, the elliptical distribution of the derived photocenter vectors might indicate opacity effects, which cause the more distant parts of the disk to appear fainter than the disk parts facing the observer. In order to include this effect in our model, we assume that the disk is embedded in a medium of constant density. After a distance $x$ traveled, the emitted intensity $I_{0}$ is then reduced to $I(x)=I_{0} \exp (-\kappa x)$. Combining this line-emission model with our best-fit continuum disk+companion model (DISK, Table 1) allows us to produce model channel maps (Figure 6, bottom), from which we compute 
line profiles, visibilities, and DPs for comparison with our data (Figure 6, top).

As line broadening mechanisms, we include thermal and turbulent Doppler broadening (Piétu et al. 2007)

$$
\Delta V=\sqrt{\frac{2 k T_{\mathrm{gas}}}{m}+v_{\mathrm{turb}}^{2}},
$$

where $v_{\text {turb }}$ is the turbulent velocity, which is typically negligible for Herbig Ae/Be stars $\left(\lesssim 0.5 \mathrm{~km} \mathrm{~s}^{-1}\right.$; e.g., Piétu et al. 2007). In the disk surface layer, the gas temperature $T_{\text {gas }}$ will be significantly higher than the dust temperature at a given stellocentric radius. We assume $T_{\text {gas }}=5 T_{\text {dust }}$, as suggested by the thermally decoupled radiative-hydrodynamics simulations by Thi et al. (2011), but note that the precise value affects mainly the line width and does not affect our general conclusions (see also Section 5.1).

Free parameters in our model are the mass of the central star $M_{A}$, the inner and outer disk radius $\left(R_{\text {in }}, R_{\text {out }}\right)$, the radial intensity power-law index $q$, the disk inclination $i$, and the opacity index $\kappa$.

We vary these parameters systematically on a parameter grid and select the best-fit model with the best $\chi_{r}^{2}=\chi_{r, V}^{2}+\chi_{r, \phi}^{2}+\chi_{r, F}^{2}$, where $\chi_{r, \phi}^{2}$ and $\chi_{r, F}^{2}$ are the reduced chi-squared between the model and measured different phase and spectrum, respectively.

Remarkably, this simple disk model can reproduce important features in our data, in particular:

1. Our model can reproduce the double-peaked $\mathrm{Br} \gamma$-line profile (Figure 6, first panel) reasonably well. One line profile characteristic that is not reproduced by our model concerns the weak asymmetry observed in $\mathrm{Br} \gamma$-line profile, with a slightly stronger redshifted line wing.

2. Our AMBER observations (Figure 6, second panel) reveal M-shaped visibility profiles on two of three baselines $\left(36.7 \mathrm{~m} / 61^{\circ}\right.$ and $\left.53.4 \mathrm{~m} / 89^{\circ}\right)$, indicating that the angular extension of the line-emitting region increases for low gas velocities. This effect is expected for a rotation-dominated velocity field, where the azimuthal velocity decreases as a function of radius and the visibility profile shape is reasonably well reproduced by our model for these baselines. On the third baseline $\left(46.6 \mathrm{~m} / 22^{\circ}\right)$, no central visibility drop has been observed. We suspect that this effect is related to more subtle radiative transfer or line broadening effects, which are not included in our simplistic model. The visibilities at low gas velocities (i.e., in the line center) would be most sensitive to such small-order effects and we leave it to future studies using full radiative transfer modeling to investigate this in more detail.

3. The measured AMBER and CRIRES DPs (Figure 6, 3rd panel) show very interesting signatures, including asymmetric S-shaped signatures and V-shaped signatures, which appear sometimes in the blueshifted and sometimes in the redshifted line wing. These signatures constrain the disk kinematics primarily, but contain also contributions from the continuum photocenter displacement caused by the companion star. Our combined binary star plus Keplerian disk rotation model reproduces the large variety of signatures reasonably well. The strongest residuals between the model and the data are observed at the longest AMBER baselines, where the data are extremely sensitive to smallorder kinematical effects, but where the $\mathrm{S} / \mathrm{N}$ is also reduced due to the lower fringe contrast.

The parameters corresponding to the best-fit model are listed in Table 2 and will be discussed in more detail in Section 5.2.
Table 2

Model-fitting Results for the VLTI/AMBER (HR-K) and VLT/CRIRES $\mathrm{Br} \gamma$-line Data (Section 4)

\begin{tabular}{lccc}
\hline \hline Parameter & & Value \\
\hline Inner emission radius & $R_{\text {in }}$ & $($ mas $)$ & $\lesssim 0.5$ \\
Outer emission radius & $R_{\text {out }}$ & $($ mas $)$ & $5.8 \pm 0.3$ \\
Position angle & $\theta$ & $\left({ }^{\circ}\right)$ & $145.0 \pm 1.3$ \\
Inclination & $i$ & $\left(^{\circ}\right)$ & $54 \pm 2$ \\
Stellar mass & $M_{\star}$ & $\left(M_{\odot}\right)$ & $(5.4 \pm 0.4) \cdot(d / 1150 \mathrm{pc})$ \\
Radial intensity index & $q$ & & $-1.0 \pm 0.1$ \\
Opacity index & $\kappa$ & & $0.5 \pm 0.2$ \\
\hline
\end{tabular}

We also investigated whether a significant fraction of the $\mathrm{Br} \gamma$ emission might be associated with the secondary star (V921 Sco B) instead of the circumprimary disk, possibly indicating active accretion from the circumbinary disk onto the secondary star. Considering the strong measured displacement of the line photocenter in the direction toward the primary (Figure 5, right) and the good quantitative agreement of the displacement vector with the flux-weighted binary astrometry vector (Section 4.1), it is clear that the secondary is not the dominant $\mathrm{Br} \gamma$-emitting component. In order to further quantify these constraints, we introduced the fraction of $\mathrm{Br} \gamma$ emission associated with the secondary star to the total $\operatorname{Br} \gamma$-flux $\left(F_{\mathrm{B}} / F_{\text {tot }}\right)_{\mathrm{Br} \gamma}$ as an additional parameter in our kinematical model and find that $\left(F_{\mathrm{B}} / F_{\text {tot }}\right)_{\mathrm{Br} \gamma}<0.11$.

\section{INTERPRETATION}

\subsection{Spectroscopy}

Our FIRE observations (Figure 7) reveal a very rich nearinfrared spectrum. Similar to the optical spectrum (Borges Fernandes et al. 2007), the near-infrared regime is dominated by strong hydrogen line emission. As listed in Table 3, we detect 27 lines from the Brackett series ( $\mathrm{Br} \gamma$ to $\mathrm{Br} 29), 24$ lines from the Paschen series ( $\mathrm{Pa} \alpha$ to $\mathrm{Pa} 24)$, and at least 10 lines from the Pfund series (Pf14-24). Given that the recombination physics of hydrogen is well known, one can use these line decrements in order to derive information about physical conditions of the line-emitting gas.

In order to model the line flux in the optically thin approximation, we extracted from Storey \& Hummer (1995) the line emissivities $\epsilon_{u l}$ for Case B recombination for all Balmer $(l=3)$, Brackett $(l=4)$, and Paschen $(l=5)$ hydrogen line transitions $(u-l)$ with $u \leqslant 25$. The line emissivities include collisional transitions, which makes our computation applicable even for relatively high gas densities. Assuming that the emitting gas is isothermal $(T=10,000 \mathrm{~K})$ and uniformly distributed in the volume $V$, the received line flux at distance $d$ is then given by

$$
F_{u-l}=\frac{N_{e} N_{p} \epsilon_{u l} V}{4 \pi d^{2}},
$$

where $N_{e}$ and $N_{p}$ are the electron and proton density per unit volume. In order to approximate the emitting volume $V$, we estimate from our $\mathrm{Br} \gamma$ spectro-interferometric data the characteristic stellocentric emission radius. For this purpose, we generate a velocity-integrated line map and measure the half-light emission radius to $r_{\text {line }}=3.0$ mas $=3.5 \mathrm{AU}$ (for $d=1.15 \mathrm{kpc}$ ). Assuming that the line-emitting gas is located in a hot disk surface layer with a characteristic scale height $h$ of $h / r_{\text {line }}=0.2$, as suggested by the thermally decoupled simulations from Thi et al. (2011), the emitting volume is given 

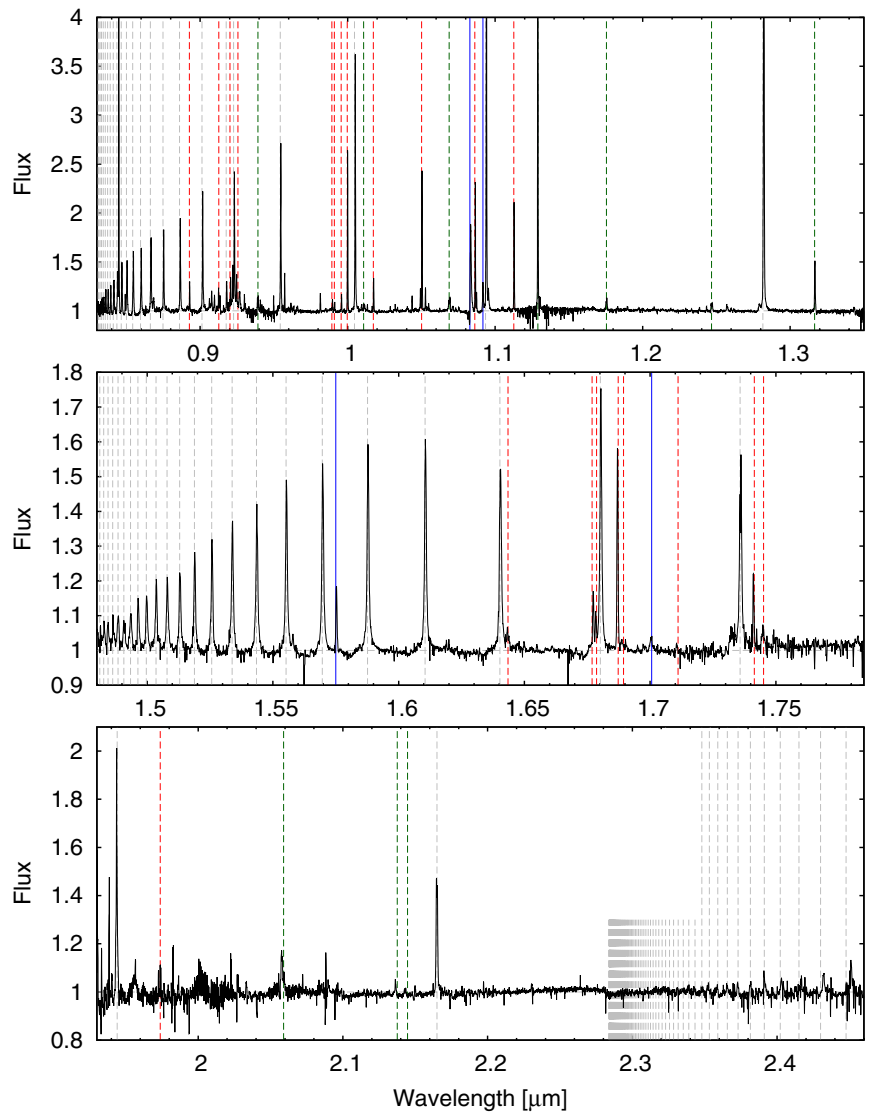

Figure 7. Near-infrared $J$-band (top), $H$-band (middle), and $K$-band (bottom) spectrum recorded with the Magellan/FIRE spectrograph. The spectrum is dominated by strong hydrogen emission lines from the Paschen, Brackett, and Pfund series (gray lines) and metallic lines from Fe II (red, dashed lines), [Fe II] (red, dotted lines), and $\mathrm{Mg}$ II, N I, Al II, C I, O I (green lines). A complete list of the identified lines can be found in Table 3. For the Pfund lines, we show besides the clearly identified transitions (Pf14-24, gray dashed lines) also the higher-order line transitions (Pf25-100, gray dotted lines) in order to mark the location of the Pfund discontinuity.

(A color version of this figure is available in the online journal.)

by $V=\pi r_{\mathrm{Br} \gamma}^{3} \cdot\left(h / r_{\text {line }}\right)$. We employ an iterative procedure, where we start by assuming a low gas density, which is then adjusted in order to reproduce the measured line fluxes.

Then we recompute the line emissivities and repeated the computation until convergence is reached. As shown in Figure 8, we require rather high gas densities between $N_{e}=N_{p}=$ $2 \times 10^{19} \mathrm{~m}^{-3}$ and $6 \times 10^{19} \mathrm{~m}^{-3}$, which supports the scenario that the gas is located in a dense disk instead of a low-density halo. For the high-level transitions ( $u>16$, i.e., Pa14-24, Br13-29, and Pf12-23), the line fluxes can be reproduced well with a single gas density $\left(N_{e}=6 \times 10^{19} \mathrm{~m}^{-3}\right)$, suggesting that the optical thin approximation is well justified. The low-level transitions $(u \leqslant 16$; i.e., $\mathrm{Pa} \beta$-Pa13, $\mathrm{Br} \gamma$-Br12) and more consistent with densities around $N_{e}=2 \times 10^{19} \mathrm{~m}^{-3}$, which might indicating that these transitions originate from more extended disk regions, where the disk surface density is lower. We would like to note that the derived densities depend to some extend also on the assumed gas temperature. Varying the temperature from the assumed $T=10,000 \mathrm{~K}$ to other realistic values (e.g., 5000-20,000 K), would change the derived densities by a factor of $\sim 2-3$. Based on our simplistic modeling, the derived density should be treated as an order-of-magnitude estimate and future radiation-hydrodynamic modeling will be required in order to fully exploit the rich information provided by our combined spectro-interferometric and spectroscopic data set.

It is interesting to compare the derived electron density to the values predicted by state-of-the-art disk models. For instance, for a typical disk around a Herbig Ae star (e.g., AB Aurigae, $2.4 M_{\odot}$ ) with a gas surface density of $10^{4} \mathrm{~kg} \mathrm{~m}^{-2}$, the predicted midplane gas density is $\sim 6 \times 10^{17} \mathrm{~m}^{-3}$ (Dullemond $\&$ Monnier 2010) at the dust sublimation radius (1.1 AU). Our density measurement of $N_{e}=(2-6) \times 10^{19} \mathrm{~m}^{-3}$ probes similar spatial scales in the V921 Sco disk and is one to two orders of magnitude higher than this value, suggesting that the disk around V921 Sco is exceptionally massive. This result is in line with the study by Henning et al. (1998), who determined the total gas mass in the millimeter cores around $25 \mathrm{Herbig} \mathrm{Ae} / \mathrm{Be}$ and FU Orionis stars and measured the highest total gas mass in the core around V921 Sco $\left(M_{\text {gas }}=40 M_{\odot}\right.$; core size: $27 \times 27^{\prime \prime}$ or $\sim 30,000 \times 30,000 \mathrm{AU}$ at $1.15 \mathrm{kpc}$ ). Observations with our spectroscopic and spectro-interferometric approach on a larger sample of protoplanetary disks will be necessary in order to determine whether the derived high gas density indicates an intrinsic property of the V921 Sco disk (possibly due to the young age of the source) or simply reflects the predicted disk surface density scaling law with stellar mass $\left(\Sigma \propto \dot{M} \propto M_{\star}^{2}\right.$; Calvet et al. 2004).

Besides hydrogen recombination lines, other identified lines include Fe II, [Fe II], C I, He I, O I, N I, Mg II, Al II, which have also been identified in the B[e] stars CI Cam (Clark et al. 1999) and HD 50138 (Jaschek et al. 1992), or in the LBV $\eta$ Car (Damineli et al. 1998; Smith \& Davidson 2001). Besides the total of 90 identified lines, we detect in our spectra a few dozen additional, yet unidentified lines and we encourage detailed follow-up spectroscopic studies in order to investigate the rich chemistry of this source.

Our wavelength range also covers the $\mathrm{CO}$ first overtone bandheads between 2.3 and $2.4 \mu \mathrm{m}$, which allows us to search for the $\mathrm{CO}$ first overtone bandheads, which we detect neither in absorption nor emission. This is particularly interesting, since Kraus (2009) suggested that these lines provide a good diagnostic tool to distinguish between a pre- and post-mainsequence evolutionary phase in $\mathrm{B}[\mathrm{e}]$ stars. According to her computation, observable ${ }^{13} \mathrm{CO}$ bandhead emission can only be produced in evolved stars, but should be absent in pre-mainsequence $B[\mathrm{e}]$ stars.

Finally, we investigated whether we find indications for variability of the $\mathrm{Br} \gamma$-line during the $\sim 6$ months covered by our CRIRES and AMBER observations. For this purpose, we convolved the CRIRES and AMBER HR-K data to the same spectral resolution and overplot the line profiles in Figure 9, finding no indications for variability, neither in line strength nor line profile.

\subsection{The Circumprimary Disk}

Using a temperature-gradient disk model (DISK), we determine the inner continuum disk radius $R_{\text {in }}$ to 1.59 mas. We can compare this value with the expected location of the dust sublimation radius, using the luminosity and distance estimate of Borges Fernandes et al. (2007), $L_{\star}=(10 \pm 3) \times 10^{3} L_{\odot}$ and $d=1150 \pm 150 \mathrm{pc}$. Assuming gray dust opacities and a standard dust sublimation temperature of $T_{\text {subl }}=1500 \mathrm{~K}$, the expected dust sublimation radius (including the effect of backwarming from the disk) is $R_{\text {subl }}=\sqrt{L_{\star} / 4 \pi \sigma T_{\text {subl }}^{4}}$ (Dullemond et al. 2001), where $\sigma$ denotes the Stefan-Boltzmann constant. With the given large luminosity and distance uncertainties, this 
Table 3

Lines Identified in Our V921 Sco FIRE Spectrum and Measured Line Equivalent Widths (EW) and Integrated Line Fluxes, $F$

\begin{tabular}{|c|c|c|c|}
\hline $\begin{array}{l}\text { Wavelength } \\
(\mu \mathrm{m})\end{array}$ & Line & $\begin{array}{l}\text { EW } \\
(\mathrm{nm})\end{array}$ & $\begin{array}{c}F \\
\left(10^{-16} \mathrm{~W} \mathrm{~m}^{-2}\right)\end{array}$ \\
\hline 0.8304 & $\mathrm{~Pa} 24$ & $-0.053^{\mathrm{a}}$ & 14.7 \\
\hline 0.8312 & $\mathrm{~Pa} 23$ & -0.073 & 20.1 \\
\hline 0.8321 & $\mathrm{~Pa} 22$ & -0.079 & 21.6 \\
\hline 0.8332 & $\mathrm{~Pa} 21$ & -0.087 & 23.7 \\
\hline 0.8343 & $\mathrm{~Pa} 20$ & -0.110 & 29.7 \\
\hline 0.8357 & Pa19 & -0.153 & 41.0 \\
\hline 0.8372 & $\mathrm{~Pa} 18$ & -0.175 & 46.4 \\
\hline 0.8390 & $\mathrm{~Pa} 17$ & -0.199 & 52.2 \\
\hline 0.8411 & Pa16 & -0.242 & 62.7 \\
\hline 0.8436 & $\mathrm{~Pa} 15$ & -0.300 & 76.6 \\
\hline 0.8447 & $\mathrm{OI}_{\mathrm{I}}$ & -1.343 & 340.4 \\
\hline 0.8465 & $\mathrm{~Pa} 14$ & -0.369 & 92.6 \\
\hline 0.8500 & Pa13 & -0.443 & 108.9 \\
\hline 0.8543 & $\mathrm{~Pa} 12$ & -0.432 & 103.7 \\
\hline 0.8596 & Pa11 & -0.339 & 79.1 \\
\hline 0.8663 & $\mathrm{~Pa} 10$ & -0.394 & 88.8 \\
\hline 0.8748 & $\mathrm{~Pa} 9$ & -0.348 & 75.3 \\
\hline 0.8820 & $\mathrm{O}_{\mathrm{I}}$ & -0.012 & 2.5 \\
\hline 0.8860 & $\mathrm{~Pa} 8$ & -0.447 & 92.2 \\
\hline 0.8927 & Fe II & -0.097 & 19.5 \\
\hline 0.9013 & $\mathrm{~Pa} 7$ & -0.462 & 89.9 \\
\hline 0.9127 & $\mathrm{Fe}$ II & -0.074 & 13.9 \\
\hline 0.9177 & Fe II & -0.169 & 31.2 \\
\hline 0.9202 & $\mathrm{Fe} I I$ & -0.121 & 22.1 \\
\hline 0.9227 & $\mathrm{~Pa} 6$ & $-0.500^{\mathrm{a}}$ & 90.8 \\
\hline 0.9256 & Fe II & $-0.080^{\mathrm{a}}$ & 14.4 \\
\hline 0.9261 & $\mathrm{OI}_{\mathrm{I}}$ & $-0.070^{\mathrm{a}}$ & 12.6 \\
\hline 0.9392 & $\mathrm{NI}_{\mathrm{I}}$ & $-0.071^{\mathrm{a}}$ & 12.3 \\
\hline 0.9406 & $\mathrm{C}_{\mathrm{I}}$ & -0.060 & 10.4 \\
\hline 0.9543 & $\mathrm{~Pa} \epsilon$ & -0.660 & 110.1 \\
\hline 0.9893 & Fe II & -0.045 & 6.9 \\
\hline 0.9910 & Fe II & -0.029 & 4.5 \\
\hline 0.9956 & $\mathrm{Fe} I I$ & -0.070 & 10.7 \\
\hline 0.9997 & $\mathrm{Fe} I \mathrm{II}$ & -0.624 & 94.4 \\
\hline 1.0047 & $\mathrm{~Pa} \delta$ & -1.132 & 169.9 \\
\hline 1.0108 & Al II & $-0.043^{\mathrm{a}}$ & 6.4 \\
\hline 1.0174 & Fe II & -0.143 & 21.0 \\
\hline 1.0501 & Fe II & -0.549 & 76.6 \\
\hline 1.0688 & $\mathrm{CI}_{\mathrm{I}}$ & -0.163 & 22.2 \\
\hline 1.0829 & He I & -0.760 & 101.7 \\
\hline 1.0862 & Fe II & -0.503 & 67.1 \\
\hline 1.0917 & He I & -0.207 & 27.4 \\
\hline 1.0935 & $\mathrm{~Pa} \gamma$ & $-1.793^{\mathrm{a}}$ & 237.2 \\
\hline 1.1127 & Fe II & -0.468 & 60.6 \\
\hline 1.1290 & $\mathrm{OI}_{\mathrm{I}}$ & $-1.874^{\mathrm{a}}$ & 238.9 \\
\hline 1.1755 & $\mathrm{C}_{\mathrm{I}}$ & -0.117 & 14.3 \\
\hline 1.2467 & $\mathrm{~N}_{\mathrm{I}}$ & $-0.083^{\mathrm{a}}$ & 9.6 \\
\hline 1.2815 & $\mathrm{~Pa} \beta$ & $-4.030^{\mathrm{a}}$ & 457.5 \\
\hline 1.3166 & $\mathrm{OI}_{\mathrm{I}}$ & -0.321 & 35.6 \\
\hline 1.4798 & Br29 & $-0.031^{\mathrm{a}}$ & 3.1 \\
\hline 1.4811 & $\operatorname{Br} 28$ & $-0.030^{\mathrm{a}}$ & 3.0 \\
\hline 1.4827 & $\mathrm{Br} 27$ & $-0.042^{\mathrm{a}}$ & 4.2 \\
\hline 1.4844 & Br26 & -0.051 & 5.1 \\
\hline 1.4863 & $\operatorname{Br} 25$ & -0.057 & 5.7 \\
\hline 1.4884 & $\mathrm{Br} 24$ & -0.072 & 7.3 \\
\hline 1.4907 & $\operatorname{Br} 23$ & $-0.075^{\mathrm{a}}$ & 7.5 \\
\hline 1.4934 & $\mathrm{Br} 22$ & -0.096 & 9.6 \\
\hline 1.4963 & $\operatorname{Br} 21$ & -0.117 & 11.7 \\
\hline 1.4997 & Br20 & -0.141 & 14.1 \\
\hline 1.5035 & Br19 & -0.161 & 16.1 \\
\hline 1.5078 & Br18 & -0.191 & 19.1 \\
\hline 1.5129 & Br17 & -0.212 & 21.1 \\
\hline 1.5188 & Br16 & -0.250 & 24.8 \\
\hline 1.5256 & Br15 & -0.279 & 27.6 \\
\hline
\end{tabular}

Table 3

(Continued)

\begin{tabular}{|c|c|c|c|}
\hline $\begin{array}{l}\text { Wavelength } \\
(\mu \mathrm{m})\end{array}$ & Line & $\begin{array}{l}\mathrm{EW} \\
(\mathrm{nm})\end{array}$ & $\begin{array}{c}F \\
\left(10^{-16} \mathrm{~W} \mathrm{~m}^{-2}\right)\end{array}$ \\
\hline 1.5338 & Br14 & -0.395 & 38.9 \\
\hline 1.5435 & Br13 & -0.430 & 42.1 \\
\hline 1.5552 & Br12 & $-0.524^{\mathrm{a}}$ & 51.0 \\
\hline 1.5696 & Br11 & -0.544 & 52.6 \\
\hline 1.5750 & Fe II & -0.105 & 10.1 \\
\hline 1.5876 & Br10 & -0.570 & 54.7 \\
\hline 1.6105 & Br9 & -0.631 & 59.9 \\
\hline 1.6403 & $\mathrm{Br} 8$ & $-0.667^{\mathrm{a}}$ & 62.5 \\
\hline 1.6435 & [Fe II] & $-0.019^{\mathrm{a}}$ & 1.8 \\
\hline 1.6769 & [Fe II] & $-0.076^{\mathrm{a}}$ & 7.0 \\
\hline 1.6787 & Fe II & $-0.046^{\mathrm{a}}$ & 4.2 \\
\hline 1.6802 & $\mathrm{Br} 7$ & $-0.719^{\mathrm{a}}$ & 66.2 \\
\hline 1.6873 & Fe II & -0.309 & 28.4 \\
\hline 1.6894 & Fe II & $-0.015^{\mathrm{a}}$ & 1.4 \\
\hline 1.7006 & He I & $-0.032^{\mathrm{a}}$ & 2.9 \\
\hline 1.7111 & {$[\mathrm{Fe} \mathrm{II}]$} & -0.013 & 1.2 \\
\hline 1.7357 & Br6 & -0.521 & 47.1 \\
\hline 1.7414 & Fe II & -0.102 & 9.2 \\
\hline 1.7451 & {$[\mathrm{Fe} \mathrm{II}]$} & $-0.054^{\mathrm{a}}$ & 4.9 \\
\hline 1.9440 & $\mathrm{Br} \delta$ & $-0.808^{b}$ & 70.3 \\
\hline 1.9738 & Fe II & -0.019 & 1.7 \\
\hline 2.0590 & Mg II & $-0.219^{\mathrm{b}}$ & 19.1 \\
\hline 2.1376 & Mg II & -0.051 & 4.5 \\
\hline 2.1447 & Mg II & -0.018 & 1.6 \\
\hline 2.1649 & $\mathrm{Br} \gamma$ & $-0.705^{\mathrm{b}}$ & 62.5 \\
\hline 2.3532 & Pf23 & -0.028 & 2.5 \\
\hline 2.3591 & Pf22 & -0.036 & 3.2 \\
\hline 2.3657 & Pf21 & -0.036 & 3.2 \\
\hline 2.3731 & Pf20 & -0.054 & 4.9 \\
\hline 2.3815 & Pf19 & -0.064 & 5.8 \\
\hline 2.3912 & Pf18 & -0.078 & 7.0 \\
\hline 2.4023 & Pf17 & -0.075 & 6.7 \\
\hline 2.4151 & Pf16 & -0.092 & 8.2 \\
\hline 2.4300 & Pf15 & -0.114 & 10.2 \\
\hline 2.4477 & Pf14 & -0.162 & 14.4 \\
\hline 2.4687 & Pf13 & -0.245 & 21.5 \\
\hline
\end{tabular}

Notes.

a These lines suffer from line blending, which introduces some uncertainty to the derived EW values.

b Due to the high $K$-band continuum flux and line brightness, these lines likely reached the nonlinearity regime of the FIRE detector, resulting in an underestimation of the real EW.

corresponds to a value of $6.9 \pm 1.1 \mathrm{AU}$ or $6.0 \pm 1.9$ mas, which is considerably larger than the measured inner disk radius of $R_{\text {in }}=1.59$ mas. Assuming higher dust sublimation temperatures of $2000 \mathrm{~K}\left(R_{\text {subl }}=3.9 \pm 0.6 \mathrm{AU}\right.$ or $\left.3.4 \pm 1.0 \mathrm{mas}\right)$ also does not solve this discrepancy. Therefore, we conclude that V921 Sco belongs to the group of Herbig Be disks that are "undersized" with respect to the size-luminosity relation (Monnier \& Millan-Gabet 2002), as already discussed by Kraus et al. (2008a) and Kreplin et al. (2012). Different physical scenarios have been proposed in order to explain this effect, including gas absorption (Monnier \& Millan-Gabet 2002), the emission from gas located inside of the dust sublimation radius (Eisner et al. 2004; Monnier et al. 2005; Kraus et al. 2008b; Tannirkulam et al. 2008), or the presence of a highly refractive dust grain species (Benisty et al. 2010). Multi-wavelength interferometric studies with even longer baseline lengths as well as detailed gas and dust radiative transfer modeling will be required in order to ultimately settle this question. 


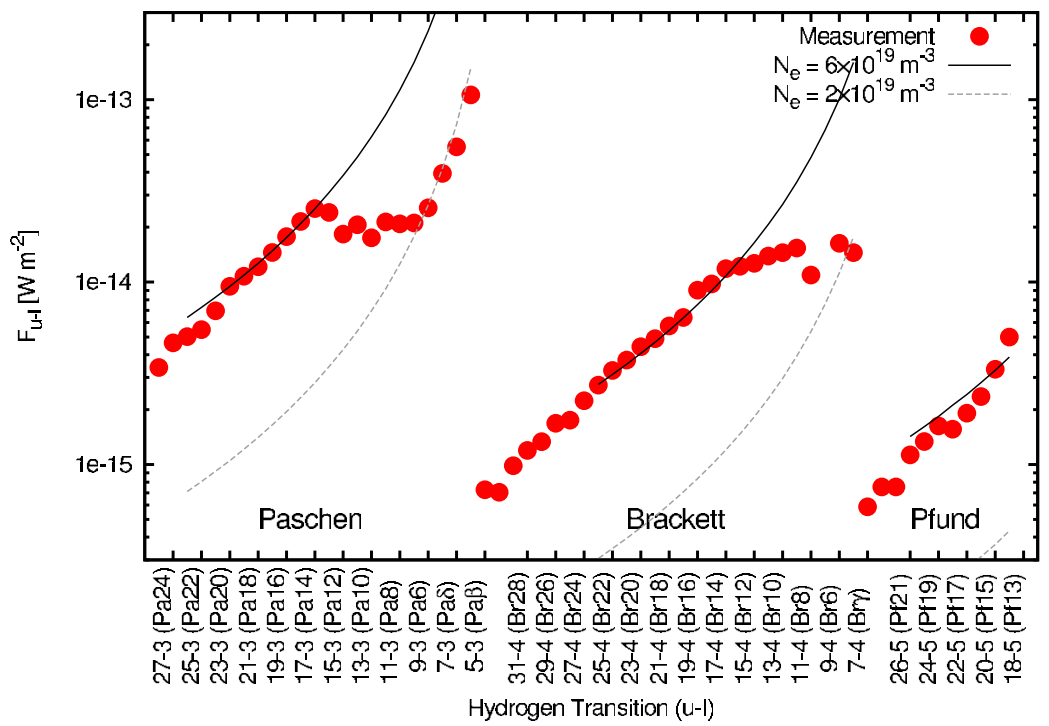

Figure 8. Line flux for the Paschen, Brackett, and Pfund decrements, as derived from our V921 Sco FIRE spectrum. The abscissa gives the upper ( $u$ ) and lower (l) levels for the corresponding hydrogen transition, where we label only about every other transition for the sake of clarity. The measured line fluxes (points) have been corrected for reddening (Section 2.3) and are overplotted with model recombination line fluxes for isothermal gas in a disk surface layer with temperature of $T=10,000 \mathrm{~K}$ and densities in the range $N_{e}=(2-6) \times 10^{19} \mathrm{~m}^{-3}$ (Section 5.1).

(A color version of this figure is available in the online journal.)

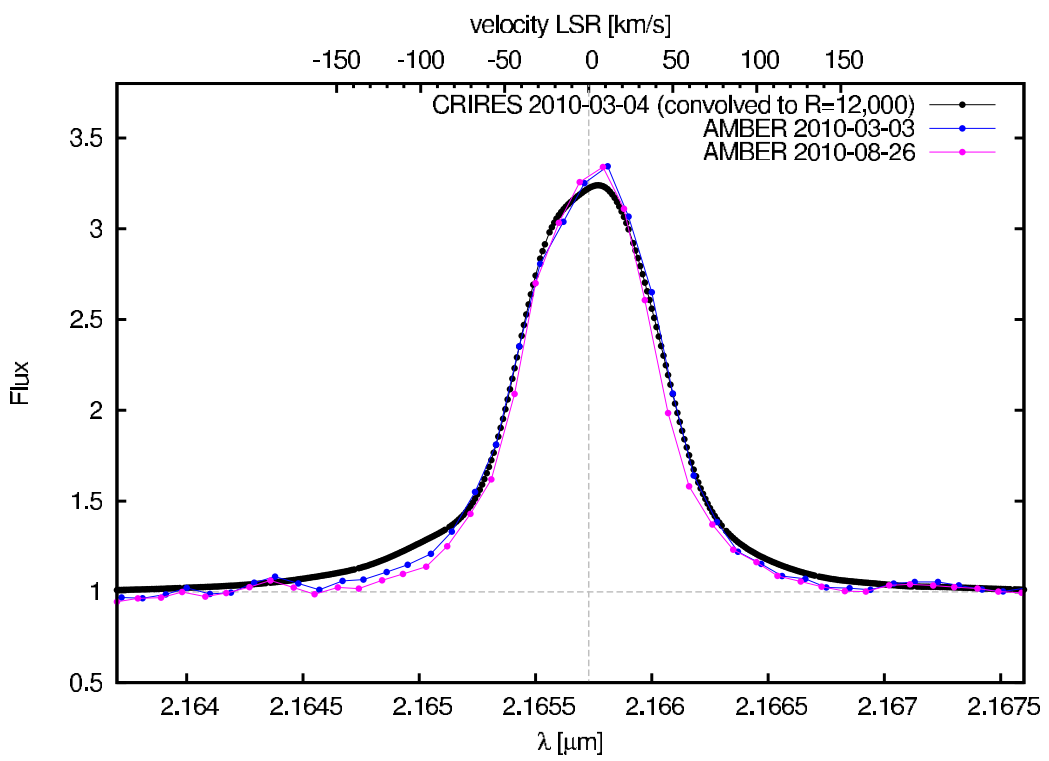

Figure 9. VLT/CRIRES and VLTI/AMBER Br $\gamma$-line spectra measured in 2010 March and August, which have been convolved to a common spectral resolution of $R=12,000$.

(A color version of this figure is available in the online journal.)

We determine the outer disk radius to $\sim 9.7$ mas. However, this value should be treated as a lower limit, since colder parts of the disk might extend to larger stellocentric radii. We can compare this derived lower limit with theoretical predictions for the disk truncation radius in binary systems. Artymowicz \& Lubow (1994) computed the resonance-induced truncation radius for circumstellar and circumbinary disks and predicts the circumprimary disk to be truncated at $r / a \sim 40 \%-20 \%$ of the orbit major axis (for a binary mass ratio of 0.3 ), depending on the orbit eccentricity and disk viscosity parameter. This range agrees reasonably well with our derived value of $R_{\text {out }} / \rho=39 \%$. For a detailed comparison, a full orbit determination and midinfrared and sub-millimeter interferometric observations of the colder material in the outer regions of the circumprimary disk and in the circumbinary gas and dust reservoir will be required.

Both theoretical (e.g., Natta et al. 2001; Dullemond et al. 2001; Isella \& Natta 2005) and observational studies (Monnier et al. 2006; Kraus et al. 2009a; Benisty et al. 2011) suggest that the inner dust rim in protoplanetary disks has a complicated, possibly puffed-up vertical structure, which can result in an asymmetric brightness distribution. Interferometric data, such as presented in this study, are able to detect these asymmetries and to constrain the corresponding disk models, in particular using phase-closure capabilities. However, it is clear that the closure phases in our AMBER data are dominated by the signatures of the newly detected companion, while potential asymmetries due to the vertical disk structure would appear 


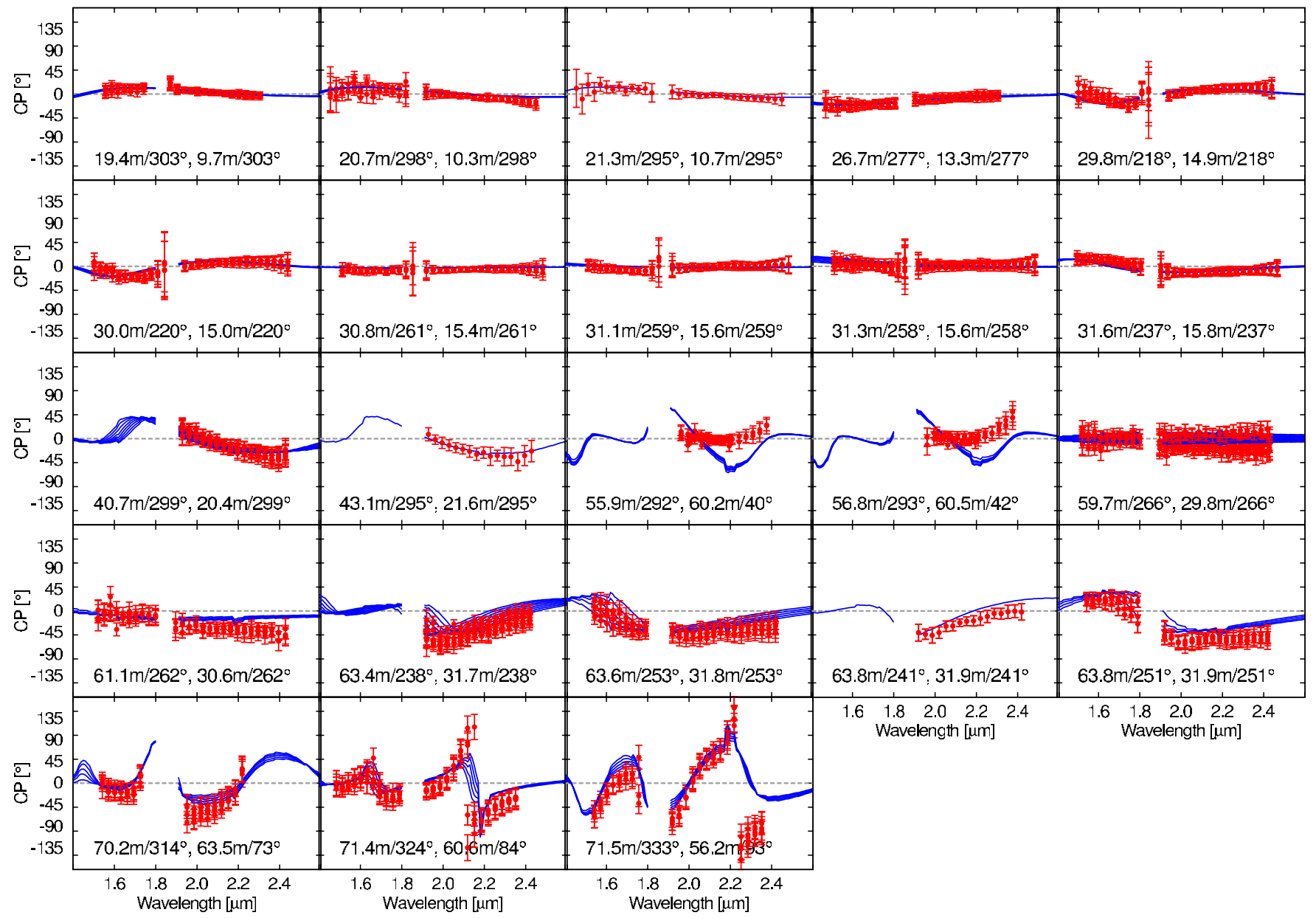

Figure 10. VLTI/AMBER closure phases and model closure phases for our best-fit model DISK. In each panel, we give the projected length and P.A. for two of the three employed baselines (the values for the third baseline are given by the closure relation).

(A color version of this figure is available in the online journal.)

only as secondary effects. Such signatures would appear in particular at long baseline lengths and close to visibility minima, where we also observe some significant deviations from our simple flat disk+companion model (Figure 10). However, it would be difficult to constrain these more complex physical models with the current uncertainties on the stellar parameters and the limited available $u v$-coverage. Therefore, we leave it to future investigations to better constrain the inner dust disk geometry around V921 Sco A. Alternatively, the remaining residuals might indicate the orbital motion of the companion within the five months during which the 2008 AMBER LR-HK data have been recorded (see Table 1 in Paper I).

Using our kinematical modeling, we show that the velocity field in the disk is in Keplerian rotation (Section 4.2) and that the line-emission emerges from a relatively high-density region $\left(N_{e}=(2-6) \times 10^{19} \mathrm{~m}^{-3}\right.$; Section 5.1), likely in a hot disk surface layer. Between the high-level $(u>16$, i.e., Pa14-24, Br13-29, and Pf12-23) and low-level transitions $(u \lesssim$ 16, i.e., $\mathrm{Pa} \beta$-Pa13, $\mathrm{Br} \gamma$-Br12), we detect a change of slope in the Paschen/Brackett decrements, indicating that the high-level transitions emerge from high-density regions closer to the star than the low-level transitions. In our best-fit model (Table 2, Figure 6), the orientation of the opacity screen is such that the southwestern part of the disk appear brighter, which suggests that the northeastern disk axis is facing toward us (which is also consistent with the orientation of the large-scale bipolar nebula,
Figures $11(\mathrm{~A})-(\mathrm{C}))$. Arguably the most surprising parameter value in our best-fit model concerns the derived stellar mass. For an assumed distance of $1.15 \mathrm{kpc}$, we yield best agreement with $5.4 \pm 0.4 M_{\odot}$, which is hardly consistent with the spectral classification $^{5}$ and the prediction mass of $9 \pm 1 M_{\odot}$ (Borges Fernandes et al. 2007).

In order to explain this surprising result, we propose two plausible scenarios.

1. The value of $1.15 \pm 0.15 \mathrm{kpc}$ (Borges Fernandes et al. 2007) might underestimate the real distance to V921 Sco. This distance has been determined based on the equivalent width of the NaI and Ca II-K interstellar absorption lines and therefore relies on a statistically established calibration. In particular, Lopes et al. (1992) employed the same method (also on the $\mathrm{Na}$ I line) and determined the distance to $2.5 \mathrm{kpc}$. A distance estimate $\gtrsim 2 \mathrm{kpc}$ has also been obtained by McGregor et al. (1988) based on optical/nearinfrared spectroscopy and photometry. Adopting such a larger distance would result in a stellar mass of $12 M_{\odot}$

\footnotetext{
5 It should be noted that the earlier spectroscopic studies were not informed about the binarity of V921 Sco, which has likely also resulted in somewhat biased spectral classifications of this intriguing source. However, given the extremely rich recombination line spectrum and the presence of He I emission lines, it seems safe to assume that the ionizing central source should be of at least B-type.
} 

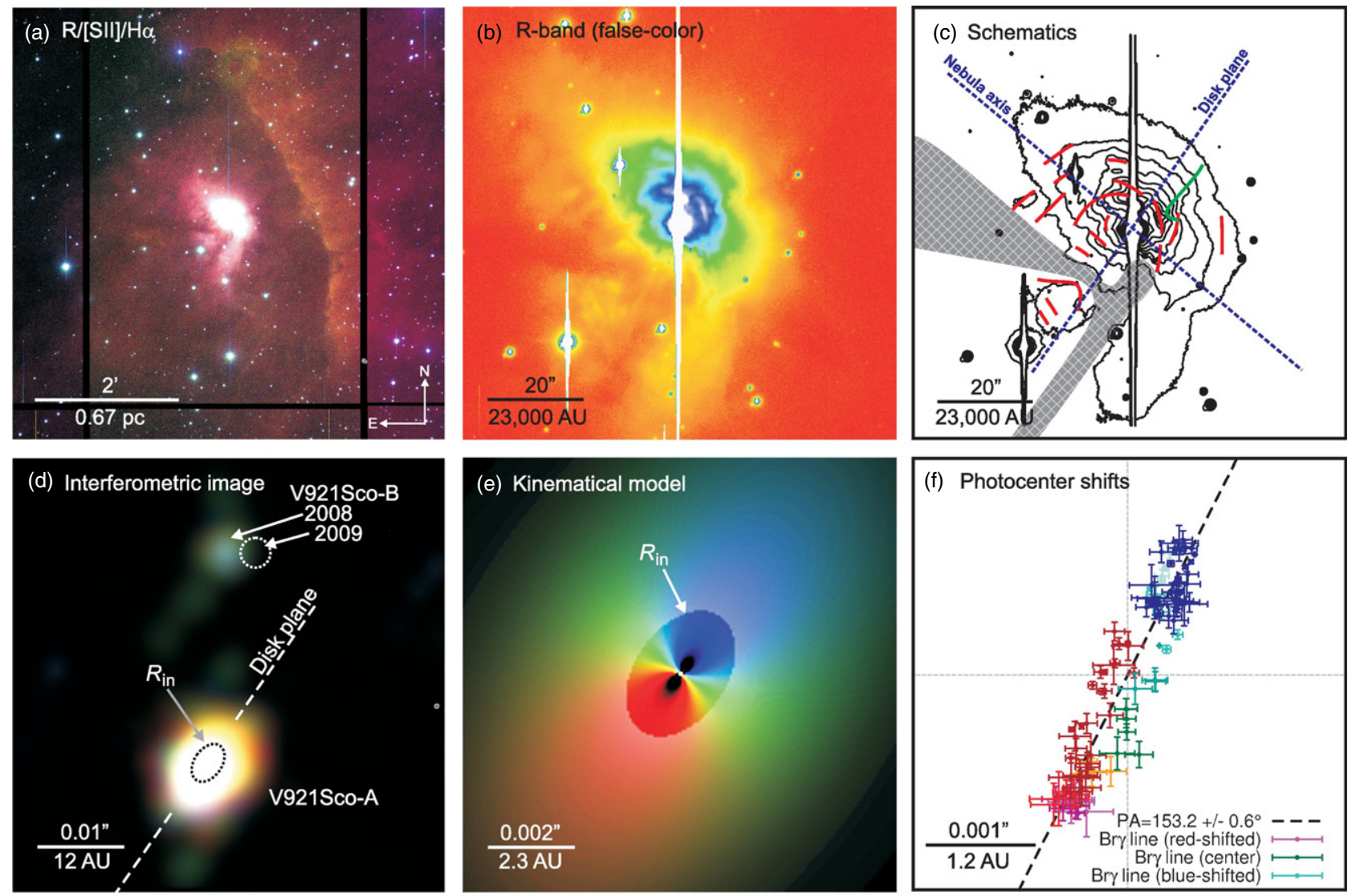

Figure 11. Zoom-in on V921 Sco, covering spatial scales over more than five orders of magnitude. On scales of a few arcminutes (A), V921 Sco is embedded in an extended ridge-like structure, as shown in this Magellan/IMACS R/[S II]/H $\alpha$ image (Paper I). On smaller scales (B), V921 Sco is surrounded by a bipolar nebula with intriguing substructure (C). The axis of the nebula is perpendicular to the plane of the disk discovered on scales of a few milliarcsecond within our interferometric image (D). The black ellipse illustrates the location of the inner continuum disk truncation radius $R_{\mathrm{in}}$, as determined with model fitting (Table 1, model DISK). Most of the $\mathrm{Br} \gamma$-line emission emerges from inside of the dust sublimation radius, as determined with our kinematical model (E), which is constrained simultaneously by the VLTI/AMBER visibilities, VLTI/AMBER+VLT/CRIRES differential phases, and the high-resolution line profile. The image shown here is a color composite of our model channel maps for gas velocities of -35 (blue), 0 (green), and $+35 \mathrm{~km} \mathrm{~s}^{-1}$ (red). The gas rotation is also revealed in a model-independent fashion by the measured photocenter offsets $(\mathrm{F})$.

(A color version of this figure is available in the online journal.)

(for $2.5 \mathrm{kpc}$ ) or $9 M_{\odot}$ (for $2 \mathrm{kpc}$ ), which would be more consistent with the spectral classification.

2. The disk might not be associated with the massive (early B-type) star in the system, but orbit around the intermediatemass (late B-type) companion instead. This would explain the relatively low derived mass, while the massive primary star could still provide sufficient ultraviolet flux to ionize the disk surface layer and the ambient low-density material resulting in the forbidden line emission. In this scenario, the accretion would occur from the circumbinary disk onto the disk around the late B-type star. The massive primary would rest closer to the center of mass and be effectively shielded from mass infall by the orbiting companion. A similar dynamical configurations seems also plausible for other Herbig B[e]-star binary systems such as MWC 361 $\mathrm{A}$, where the active accretion is clearly associated with the less massive component in the system (Monnier et al. 2006; Alecian et al. 2008).

In order to distinguish between these scenarios, it will be necessary to accurately measure the flux ratio of the two stars as a function of wavelength. Our current measurement yield similar flux ratios between the two stars in the $H$ and $K$ bands, with some indications that V921 Sco A (i.e., the star associated with the circumstellar material) is of earlier spectral type (Paper I), favoring scenario (1). However, it should be noted that this color measurement is only significant at the $2 \sigma$ level $\left(\left(F_{\mathrm{B}} / F_{\mathrm{A}}\right)_{H}=0.83 \pm 0.15,\left(F_{\mathrm{B}} / F_{\mathrm{A}}\right)_{K}=1.18 \pm 0.12\right.$, Paper I $)$ and might be biased, for instance in case of inhomogeneous extinction toward the two stars. In both scenarios, it is clear that a careful re-evaluation of the spectral classification based on the spectral energy distribution and the spectroscopic diagnostics will be necessary, but this is out of the scope of this paper.

Independent of these uncertainties, we can derive new insights on the evolutionary status of V921 Sco from our constraints on the disk gas velocity field. The decretion disks around postmain-sequence supergiant $\mathrm{B}[\mathrm{e}]$ stars should exhibit a strong outflowing velocity component (Lamers \& Pauldrach 1991), which is not observed in our data. On the other hand, our observation of a Keplerian rotation profile is consistent with the expected velocity field in a viscous accretion disk (Shakura \& Sunyaev 1973) around a pre-main-sequence Herbig B[e] star.

\subsection{On the Origin of the $\mathrm{Br} \gamma$ emission in Herbig Be Stars}

Arguably, the most important advancement provided by our observational data set on V921 Sco is that it allows us to simultaneously constrain the geometry of the circumstellar dust disk and the spatial distribution and kinematics of the hot gas in the system (see Figure 6). Such constraints are 
important, in particular as earlier spatially unresolved studies have associated the origin of the $\mathrm{Br} \gamma$-line emission with fundamentally different physical mechanisms, including mass infall (e.g., magnetospheric accretion, gaseous inner disks) and mass outflow processes (e.g., stellar winds, X-winds, or disk winds). For V921 Sco, we find that the $\mathrm{Br} \gamma$-emitting gas clearly shows a rotation-dominated velocity field and orbits the star in the same plane $\left(\varphi=145.0 \pm 1.3, i=54 \pm 2^{\circ}\right.$, see Table 2$)$ as the circumstellar dust disk $\left(\varphi=145^{\circ} .0 \pm 8.4\right.$, $i=48.8 \pm 4^{\circ}$ ), as illustrated in Figure 11. On the other hand, Benisty et al. (2010) found for the Herbig Be-star component in the $\mathrm{Z} \mathrm{CMa}$ binary system that the $\mathrm{Br} \gamma$-line emission was displaced along the outflow axis, i.e., presumably perpendicular to the disk plane. Given that the $\mathrm{Z}$ CMa observations were obtained during an exceptionally strong outbursting phase of the Z CMa system in 2008, they likely do not represent typical conditions in a Herbig Be star. Another example is provided by MWC 297, where Weigelt et al. (2011) could reproduce important features of the measured wavelengthdifferential phases using a magnetospheric disk wind model. These examples, V921 Sco (where $\mathrm{Br} \gamma$ was found to trace orbiting gas in the disk plane), $\mathrm{Z} \mathrm{CMa} \mathrm{(where} \mathrm{the} \mathrm{Br} \gamma$-emitting gas was observed in a bipolar wind), and MWC 297 (where the $\mathrm{Br} \gamma$-emitting might trace a disk wind), illustrate that the $\mathrm{Br} \gamma$ emission is probably not associated with a single astrophysical process, but can trace both mass accretion and outflow processes, as we have already concluded from our small VLTI/AMBER $\mathrm{Br} \gamma$-survey with spectral resolution $R=1500$ (Kraus et al. 2008a). Spatially and spectrally resolved observations on a larger object sample will be essential in order to explore under which circumstances the $\mathrm{Br} \gamma$-line traces a certain mechanism and to determine the relation with spectroscopic diagnostics.

\section{CONCLUSIONS}

In this paper, we have investigated the milliarcsecond-scale environment around the B[e] star V921 Sco and constrained the spatial distribution and kinematics of ionized hydrogen gas in the system with a spectral resolution up to $R=100,000$. We summarize our findings as follows.

1. Our $H$ - and $K$-band continuum interferometric images reveal a spatially extended (Gaussian FWHM 7.5 mas) disklike structure, seen under an intermediate inclination angle of $\sim 48.8 \pm 4^{\circ}$. Using a temperature-gradient model, we determine the dust sublimation radius to be $1.59 \pm 0.25$ mas and find that the apparent disk size increases with wavelength, consistent with an irradiated dust disk.

2. Our VLTI/AMBER $(R=12,000)$ and VLT/CRIRES ( $R=100,000)$ observations spatially and spectrally resolve the $\mathrm{Br} \gamma$-line emission from V921 Sco on submilliarcsecond scales. Using a model-independent photocenter analysis technique and our detailed kinematical modeling, we find that the line-emitting gas is located in a Keplerian-rotating disk, which extends down to a few stellar radii. We interpret this finding as strong evidence for the pre-main-sequence nature of the object, since the decretion disks in post-main-sequence $\mathrm{B}[\mathrm{e}]$-stars are believed to exhibit a significant outflowing velocity component.

3. From our disk kinematical modeling, we derive a mass of $5.4 \pm 0.4 M_{\odot} \cdot(d / 1150 \mathrm{pc})$ for the central object. Assuming the distance of $1.15 \mathrm{kpc}$ proposed by Borges Fernandes et al. (2007), we find that this mass is too low to be consistent with the early B-type spectral classification, which might indicate that the distance to V921 Sco is probably considerably larger $(\sim 2 \mathrm{kpc})$. Alternatively, the disk might be associated with the less massive component in the system. In this dynamical scenario, the material from the circumbinary disk is accreted onto the intermediate-mass (late B-type) component, preventing significant accretion onto the early B-type star. In any case, a careful reevaluation of the spectral classification of the components in this enigmatic binary system will be required in future studies.

4. Our FIRE observations reveal a rich near-infrared emission line spectrum of permitted and forbidden emission lines (Fe II, [Fe II], Ci, He I, O I, N I, Mg II, Al II), but show no sign of $\mathrm{CO}$ bandhead emission, which supports a pre-mainsequence nature (Kraus 2009).

5. From the FIRE spectra, we derive the line flux for 61 hydrogen recombination lines, including transitions from the Paschen, Brackett, and Pfund series. Utilizing the spatial information provided by our VLTI/AMBER spectrointerferometric data allows us to model the line decrements with a hydrogen excitation model and to derive electron densities for the line-emitting gas. The derived high number densities $\left(N_{e}=(2-6) \times 10^{19} \mathrm{~m}^{-3}\right)$ suggest that the disk around V921 Sco is particularly massive, reinforcing the conclusion of Henning et al. (1998), who derived a total gas mass of $40 M_{\odot}$ for the surrounding millimeter core.

Our combined spectro-interferometry and line decrement modeling approach provides a novel tool to estimate the gas density in the innermost AU of protoplanetary disks. Applying this tool on a larger sample of $\mathrm{T}$ Tauri, Herbig Ae/Be, and transitional disks might allow, for instance, to quantify gas depletion as a function of disk evolution, or to confirm the expected relations between the mass accretion rate, disk surface density, and stellar mass (e.g., Calvet et al. 2004).

Furthermore, our study demonstrates the power of combining infrared spectro-interferometry with the technique of spectroastrometry. Spectro-interferometry is indispensable to characterize the continuum geometry (measurement of the dust disk geometry and, for instance, the detection of companions) and to resolve the detailed geometry and kinematics of the circumstellar gas. On the other hand, spectro-astrometry provides a resource-efficient and straightforward method to measure first-order kinematical information with very high spectral dispersion in the spatially unresolved regime, providing highly complementary constraints for the interpretation of spectrointerferometric signatures.

We thank the anonymous referee for constructive comments, which helped to improve the manuscript. This work was done in part under contract with the California Institute of Technology (Caltech), funded by NASA through the Sagan Fellowship Program (S.K. is a Sagan Fellow).

Facilities: VLTI, VLT:Antu, Magellan:Clay

\section{REFERENCES}

Acke, B., \& van den Ancker, M. E. 2006, A\&A, 457, 171

Acke, B., van den Ancker, M. E., \& Dullemond, C. P. 2005, A\&A, 436, 209

Alecian, E., Catala, C., Wade, G. A., et al. 2008, MNRAS, 385, 391

Artymowicz, P., \& Lubow, S. H. 1994, ApJ, 421, 651

Bailey, J. 1998a, MNRAS, 301, 161

Bailey, J. A. 1998b, Proc. SPIE, 3355, 932

Benedettini, M., Nisini, B., Giannini, T., et al. 1998, A\&A, 339, 159

Benisty, M., Malbet, F., Dougados, C., et al. 2010, A\&A, 517, L3 
Benisty, M., Renard, S., Natta, A., et al. 2011, A\&A, 531, A84

Borges Fernandes, M., Kraus, M., Lorenz Martins, S., \& de Araújo, F. X. 2007, MNRAS, 377, 1343

Brannigan, E., Takami, M., Chrysostomou, A., \& Bailey, J. 2006, MNRAS, 367, 315

Calvet, N., Muzerolle, J., Briceño, C., et al. 2004, AJ, 128, 1294

Cardelli, J. A., Clayton, G. C., \& Mathis, J. S. 1989, ApJ, 345, 245

Chelli, A., Utrera, O. H., \& Duvert, G. 2009, A\&A, 502, 705

Cidale, L., Zorec, J., \& Tringaniello, L. 2001, A\&A, 368, 160

Clark, J. S., Steele, I. A., Fender, R. P., \& Coe, M. J. 1999, A\&A, 348, 888

Damineli, A., Stahl, O., Kaufer, A., et al. 1998, A\&AS, 133, 299

de Winter, D., \& The, P. S. 1990, Ap\&SS, 166, 99

Dullemond, C. P., Dominik, C., \& Natta, A. 2001, ApJ, 560, 957

Dullemond, C. P., \& Monnier, J. D. 2010, ARA\&A, 48, 205

Eisner, J. A., Lane, B. F., Hillenbrand, L. A., Akeson, R. L., \& Sargent, A. I. 2004, ApJ, 613, 1049

Gai, M., Menardi, S., Cesare, S., et al. 2004, Proc. SPIE, 5491, 528

Goto, M., Carmona, A., Linz, H., et al. 2012, ApJ, 748, 6

Habart, E., Testi, L., Natta, A., \& Vanzi, L. 2003, A\&A, 400, 575

Hase, F., Wallace, L., McLeod, S. D., Harrison, J. J., \& Bernath, P. F. 2010, J. Quantum Spectrosc. Radiat. Transfer, 111, 521

Henning, T., Burkert, A., Launhardt, R., Leinert, C., \& Stecklum, B. 1998, A\&A, 336, 565

Hillenbrand, L. A., Strom, S. E., Vrba, F. J., \& Keene, J. 1992, ApJ, 397, 613

Hutsemekers, D., \& van Drom, E. 1990, A\&A, 238, 134

Isella, A., \& Natta, A. 2005, A\&A, 438, 899

Jaschek, M., Jaschek, C., Andrillat, Y., \& Houziaux, L. 1992, MNRAS, 254, 413

Kaeufl, H., Ballester, P., Biereichel, P., et al. 2004, Proc. SPIE, 5492, 1218

Kraus, M. 2009, A\&A, 494, 253

Kraus, S., Calvet, N., Hartmann, L., et al. 2012a, ApJ, 746, L2

Kraus, S., Hofmann, K.-H., Benisty, M., et al. 2008a, A\&A, 489, 1157

Kraus, S., Hofmann, K.-H., Malbet, F., et al. 2009a, A\&A, 508, 787

Kraus, S., Monnier, J. D., Che, X., et al. 2012b, ApJ, 744, 19

Kraus, S., Preibisch, T., \& Ohnaka, K. 2008b, ApJ, 676, 490
Kraus, S., Weigelt, G., Balega, Y. Y., et al. 2009b, A\&A, 497, 195 Kreplin, A., Kraus, S., Hofmann, K.-H., et al. 2012, A\&A, 537, A103 Lachaume, R. 2003, A\&A, 400, 795

Lamers, H. J. G. L. M., \& Pauldrach, A. W. A. 1991, A\&A, 244, L5

Lamers, H. J. G. L. M., Zickgraf, F., de Winter, D., Houziaux, L., \& Zorec, J. 1998, A\&A, 340, 117

Le Bouquin, J., Bauvir, B., Haguenauer, P., et al. 2008, A\&A, 481, 553

Lopes, D. F., Damineli Neto, A., \& de Freitas Pacheco, J. A. 1992, A\&A, 261, 482

McGregor, P. J., Hyland, A. R., \& Hillier, D. J. 1988, ApJ, 324, 1071

Mérand, A., Bordé, P., \& Coudé Du Foresto, V. 2006, A\&A, 447, 783

Monnier, J. D., Berger, J.-P., Millan-Gabet, R., et al. 2006, ApJ, 647, 444

Monnier, J. D., \& Millan-Gabet, R. 2002, ApJ, 579, 694

Monnier, J. D., Millan-Gabet, R., Billmeier, R., et al. 2005, ApJ, 624, 832

Natta, A., Palla, F., Butner, H. M., Evans II, N. J., \& Harvey, P. M. 1993, ApJ, 406, 674

Natta, A., Prusti, T., Neri, R., et al. 2001, A\&A, 371, 186

Petrov, R. G., Malbet, F., Weigelt, G., et al. 2007, A\&A, 464, 1

Piétu, V., Dutrey, A., \& Guilloteau, S. 2007, A\&A, 467, 163

Pontoppidan, K. M., Blake, G. A., \& Smette, A. 2011, ApJ, 733, 84

Shakura, N. I., \& Sunyaev, R. A. 1973, A\&A, 24, 337

Shore, S. N., Brown, D. N., Bopp, B. W., et al. 1990, ApJS, 73, 461

Simcoe, R. A., Burgasser, A. J., Bernstein, R. A., et al. 2008, Proc. SPIE, 7014, $70140 \mathrm{U}$

Smith, N., \& Davidson, K. 2001, ApJ, 551, L101

Štefl, S., Rivinius, Th., Carciofi, A. C., et al. 2009, A\&A, 504, 929

Storey, P. J., \& Hummer, D. G. 1995, MNRAS, 272, 41

Takami, M., Bailey, J., \& Chrysostomou, A. 2003, A\&A, 397, 675

Tannirkulam, A., Monnier, J. D., Millan-Gabet, R., et al. 2008, ApJ, 677, L51

Tatulli, E., Isella, A., Natta, A., et al. 2007a, A\&A, 464, 55

Tatulli, E., Millour, F., Chelli, A., et al. 2007b, A\&A, 464, 29

The, P. S., de Winter, D., \& Perez, M. R. 1994, A\&AS, 104, 315

Thi, W., Woitke, P., \& Kamp, I. 2011, MNRAS, 412, 711

Weigelt, G., Grinin, V. P., Groh, J. H., et al. 2011, A\&A, 527, A103

Whelan, E. T., Ray, T. P., Bacciotti, F., et al. 2005, Nature, 435, 652 